San Jose State University

SJSU ScholarWorks

Master's Theses

Master's Theses and Graduate Research

Summer 2020

\title{
Understanding The Role of Eddy Vorticity Fluxes On The Rapid Intensification of Hurricane Irma(2017) And Michael (2018)
}

Alrick L. Green

San Jose State University

Follow this and additional works at: https://scholarworks.sjsu.edu/etd_theses

\section{Recommended Citation}

Green, Alrick L., "Understanding The Role of Eddy Vorticity Fluxes On The Rapid Intensification of Hurricane Irma(2017) And Michael (2018)" (2020). Master's Theses. 5124.

DOI: https://doi.org/10.31979/etd.t8fw-xtee

https://scholarworks.sjsu.edu/etd_theses/5124

This Thesis is brought to you for free and open access by the Master's Theses and Graduate Research at SJSU ScholarWorks. It has been accepted for inclusion in Master's Theses by an authorized administrator of SJSU ScholarWorks. For more information, please contact scholarworks@sjsu.edu. 
UNDERSTANDING THE ROLE OF EDDY VORTICITY FLUXES ON THE RAPID INTENSIFICATION OF HURRICANE IRMA (2017) AND MICHAEL (2018)

A Thesis

Presented to

The Faculty of the Department of Meteorology and Climate Science

San José State University

In Partial Fulfillment

of the Requirements for the Degree

Master of Science

by

Alrick L. Green

August 2020 
(C) 2020

Alrick L. Green

ALL RIGHTS RESERVED 
The Designated Thesis Committee Approves the Thesis Tilted

UNDERSTANDING THE ROLE OF EDDY VORTICITY FLUXES ON THE RAPID INTENSIFICATION OF HURRICANE IRMA (2017) AND MICHAEL (2018)

\author{
by \\ Alrick L. Green \\ APPROVED FOR THE DEPARTMENT OF METEOROLOGY AND CLIMATE \\ SCIENCE
}

SAN JOSÉ STATE UNIVERSITY

August 2020

Sen Chiao, Ph.D. (Chair)

Department of Meteorology \& Climate Science

Sundararaman Gopalakrishnan, Ph.D. Lead Meteorologist, Modeling and HFIP

Development Manager, NOAA/AOML/

Hurricane Research Division

Ghassan Alaka, Jr, Ph.D.

IT Specialist, NOAA/AOML/Hurricane

Research Division 


\section{DEDICATION}

To each and every person that has walked in and out of my life.

In loving memory of two very special friends...

Yohance Frederick Fegan

(07/30/1991- 03/06/2013)

Marienette Fotsing (Ndemnye) Ngambou

$(07 / 02 / 1992-05 / 21 / 2015)$

"Life is about eating good food and laughter."

-Dennis Green- (My father)

"The journey is never ending. There's always gonna be growth, improvement, adversity; you just gotta take it all in and do what's right, continue to grow, continue to live in the moment."

-Antonio Brown- 


\begin{abstract}
UNDERSTANDING THE ROLE OF EDDY VORTICITY FLUXES ON THE RAPID INTENSIFICATION OF HURRICANE IRMA (2017) AND MICHAEL (2018)
\end{abstract}

\begin{abstract}
by Alrick L. Green
Rapid Intensification (RI) in TCs still remains one of the most challenging tasks for forecasters. The lack of understanding of the physical mechanisms and complexities in charge of these events, limits our understanding and makes it more difficult for weather models to resolves and forecast RI events. The Hurricane Weather Research and Forecasting (HWRF) model was used to examine the connection between rapid intensification and environmental forcing for Hurricane Irma (2017) and Hurricane Michael (2018). The tangential wind momentum budget was used for a comparative analysis for these two cases to identify similarities and differences in the pathways to intensification in varied environments. The eddy radial vorticity flux term is a focal point as previous studies have found it to have a significant role in intensity change and vortex spin up in sheared tropical cyclones. Our findings based on this work shows that eddy vorticity fluxes negatively impacted the vortex spin up and intensification in both cases. Despite the persistent environmental shear associated with Hurricane Michael, the mean transport of horizontal and vertical momentum was the dominating factor in spin up of the vortex from the bottom up.
\end{abstract}




\section{ACKNOWLEDGEMENTS}

I would first and foremost like to thank the grace of God for guiding me through this journey throughout my studies. I would like to thank the Lord for giving me the strength, patience, motivation, and the will to continue on while facing hard times throughout my program and my life. Special thanks to my family and friends for their continuous prayers, motivational speeches, support, and unconditional love. I could not have done it with you all. A special thank you to the National Oceanic and Atmospheric Administration (NOAA), Educational Partnership Program (EPP), U.S. Department of Commerce, under Agreement No. NA16SEC4810006 and the NOAA Center for Atmospheric Science and Meteorology (NCAS-M) at Howard University for supporting me as a NCAS-M fellow student.

Also, the Center for Applied Atmospheric Research and Education (CAARE) at San José State University. A special thank you to my mentors, Drs. Sundararaman Gopalakrishnan, Ghassan Alaka Jr, Xuejin Zhang, and the Hurricane Research Division for their guidance and input throughout my internship. Lastly, the greatest thank you goes to my advisor, Dr. Sen Chiao. Thank you so much for having an immense amount of patience and all the help you have given me throughout my entire graduate school career to succeed. I could not have done it if it was not for your generosity, strong work ethics, mentorship, and believing in me. 


\section{TABLE OF CONTENTS}

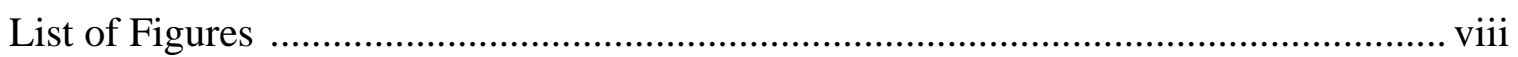

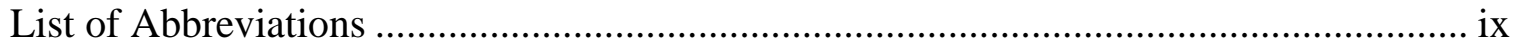

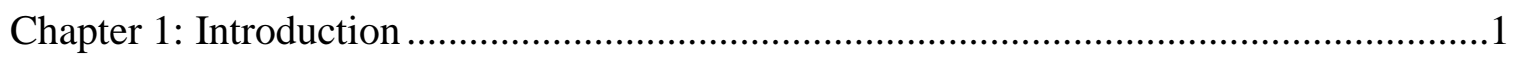

Chapter 2: Research Question, Objectives, and Hypotheses .............................................5

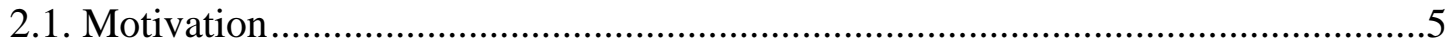

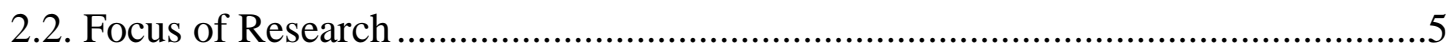

2.3. Hypotheses ................................................................................................

Chapter 3: Case Description. Experimental Design, and Methods......................................

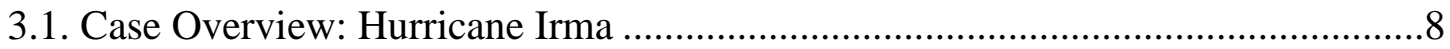

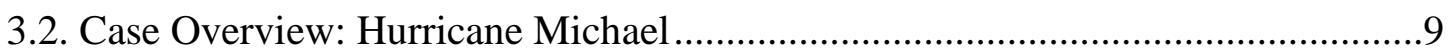

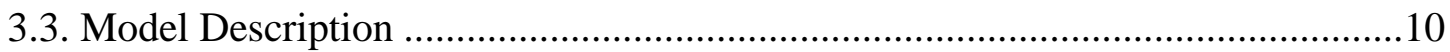

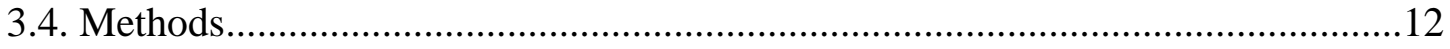

3.5. Tangential Wind Momentum Budget Terms ………………….............................13

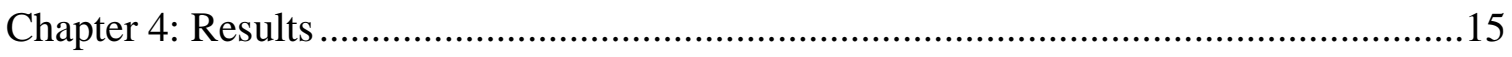

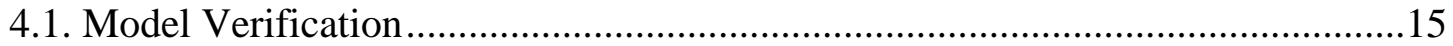

4.2. Large Scale Wind Shear..................................................................................20

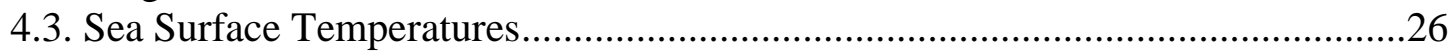

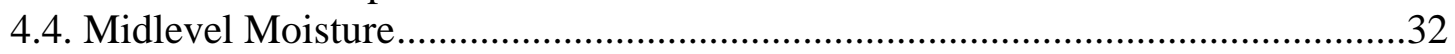

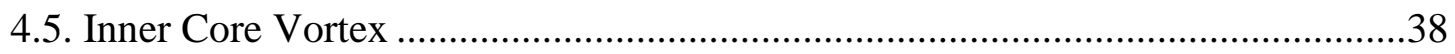

4.6. Tangential Wind Momentum Budgets ................................................................44

Chapter 5: Conclusions and Discussions .................................................................54

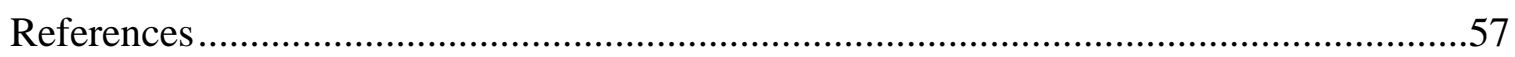




\section{LIST OF FIGURES}

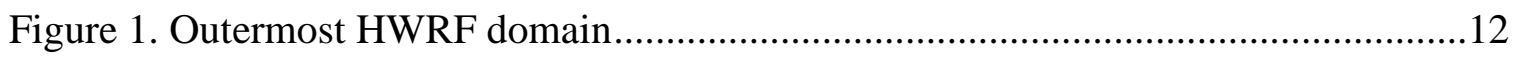

Figure 2. Tangential wind momentum budgets equation...........................................14

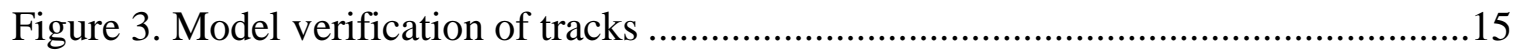

Figure 4. Model verification of central minimum pressures and maximum winds ...........17

Figure 5. 850-200 hPa shear (shaded) and 200-hPa streamlines for Irma ......................22

Figure 6. 850-200 hPa shear (shaded) and 200-hPa streamlines for Michael ..................25

Figure 7. Sea surface temperatures and pressure contours for Irma ..............................28

Figure 8. Sea surface temperatures and pressure contours for Michael ...........................31

Figure 9. Midlevel moisture and pressure contours for Irma.......................................34

Figure 10. Midlevel moisture and pressure contours for Michael ................................37

Figure 11. Azimuthal structure of vertical velocity and vorticity for Irma ....................40

Figure 12. Azimuthal structure of vertical velocity and vorticity for Michael .................43

Figure 13. Tangential wind momentum budget for Irma early RI...............................46

Figure 14. Tangential wind momentum budget for Irma late RI ...............................48

Figure 15. Tangential wind momentum budget for Michael early RI ...........................51

Figure 16. Tangential wind momentum budget for Michael late RI ..............................53 


\section{LIST OF ABBREVIATIONS}

AOML -The Atlantic Oceanographic and Meteorological Laboratories

CBs - Convective Bursts

ERCs- Eyewall Replacement Cycles

EMC - Environmental Modeling Center

GDAS -Global Data Assimilation System

GFDL - The Geophysical Fluid Dynamics Laboratory

GFS -Global Forecast System

HFIP -Hurricane Forecast Improvement Project

HRD -Hurricane Research Division

HWRF -Hurricane Weather Research and Forecasting

Km -Kilometers

LSM -The Noah Land Surface Model

MDR -Main Development Region

MPIPOM-TC -Message Passing Interface Princeton Ocean Model-Tropical Cyclone

NCEP -National Center for Environmental Prediction

NI -Non Intensifying

NOAA -National Oceanographic and Atmospheric Administration

NWS -National Weather Service

PBL -Planetary Boundary Layer

RI -Rapid Intensification

RMW -Radius of Maximum Winds

SST/SSTs -Sea Surface Temperature(s)

TC -Tropical Cyclone

TS-Tropical Storm 



\section{INTRODUCTION: RAPID INTENSIFICATION OF TROPICAL CYCLONE IN SHEARED ENVIRONMENTS}

A tropical cyclone (TC) is one of the most dangerous forms of natural disaster to impact society and coastal communities annually. Thus, it is very important to accurately predict in advance the track and changes in intensity associated with these weather systems. In addition, forecasting rapid intensification (RI, a change in maximum wind speed of 30 knots $(15 \mathrm{~m} / \mathrm{s})$ or more in a 24 hour period, Kaplan et al. 2010), especially in asymmetric TCs still remains one of the most challenging tasks for forecasters. The lack of understanding of the physical mechanisms and complexities in charge of these events, makes it difficult for operational meteorologists to forecast RI events (Kaplan and DeMaria 2003; Kaplan et al. 2010). A recent study by Rappaport et al. (2009) reports that modern dynamical tropical cyclone models still underperform and have not acquired statistical intensity models skill levels due to a bias of underestimation or overestimation of TC intensities. These types of biases prevail due to the inability to accurately resolve key features such as spiral rain bands, and polygon eyes wall during evolution of the inner core vortex.

In addition, TC intensity variability involves multiscale nonlinear interaction of different variables and phenomena (Marks et al. 1998; Shen et al. 2010). These challenges are due to multiscale interaction of environmental factors such as vertical wind shear, (Gray 1968; Merrill 1988; DeMaria and Kaplan 1994; Chen and Gopalakrishnan 2015), ample midlevel moisture (Gray 1968; Chen and Gopalakrishnana 2015), upper ocean temperatures (Byers 1944; Black 1983; Shay et al. 2010), cloud 
microphysics, air sea interaction, and inner core dynamics and thermodynamics (Schubert and Hack 1982; Willoughby et al. 1982; Kossin and Schubert 2001; Eastin et al. 2005a,b) which are well known to influence the RI of tropical cyclones.

Vertical wind shear is widely recognized to be one of the most pressing issues in regards to forecasting TC's intensification (DeMaria 1996; Kaplan and DeMaria 2003; Zhang and Tao 2013; Kaplan et al. 2015). Environmental shear interaction with TC vortex has been greatly studied by both observation and numerical simulations. Several proposed mechanisms have been identified to explain the negative impacts responsible for shear-induced disparities of TC intensity evolution. A few of these influences are: vortex tilting (DeMaria 1996; Kaplan and DeMaria 2003; Kaplan et al. 2010; Balachandran et al. 2019), midlevel ventilation of the inner core (Tang and Emanuel 2010), and shear induced dry-air into the storm's inner core region that causes a reduction of moist entropy important for intensification (Riemer et al. 2010).

Recent studies emphasized the complexities associated with linking the relationship between environmental shear and TC intensity. There have been a few observed cases of tropical cyclones intensifying under hostile environmental shear. Chen and Gopalakrishnan (2015) investigated the rapid intensification of Hurricane Earl (2010) in a sheared environment using the operational Hurricane Weather Research and Forecasting (HWRF) system. In this study, a great number of aircraft observations of the inner core were used to verify the forecast of Hurricane Earl to understand the asymmetric RI under hostile environments. The researchers found that the triggering mechanism for RI was the development of an upper level warm core in the center of the vortex. This core developed 
due to upshear convection along with upshear warm advection caused by subsidence warming in the upshear left region. This warming moved radially inwards from the upshear left region towards the lower level center of the vortex (Chen and Gopalakrishnan 2015).

Another case of TC experienced RI under hostile conditions is Tropical Storm Gabrielle (2001). Tropical Storm Gabrielle was within an area of strong environmental shear of $13 \mathrm{~m} / \mathrm{s}$ when it underwent RI after the storm's central pressure dropped to $22 \mathrm{hPa}$ within 3 hours. Molinari et al (2006) and Molinari and Vallaro (2010) results from this study, found that the key factor behind Gabrielle RI despite high environmental shear was an intense convective cell. This intense convective cell developed in the downshear left quadrant of the vortex and moved cyclonically inward, allowing for amplification of kinetic energy production to spin up the vortex (Chen and Gopalakrishnan 2015).

Rios-Berrios et al.(2016a) examined the rapid intensification of Hurricane Katia (2011) in a sheared environment. This study found that, TC intensification occurred due to the moist environment located opposite of the sheared region and was responsible for the RI event despite the presence of environmental shear. Zhang and Tao (2013) found that vertical shear increases the uncertainty of TC intensity forecast. Bhatia and Nolan (2013) demonstrated that TC intensity forecast models of initial prediction of strong TCs in moderate shear produced some of the largest intensity forecast errors.

Leighton et al. (2018) conducted a study using Hurricane Weather Research and Forecasting (HWRF) ensemble experimental forecasts to investigate the rapid intensification process associated with Hurricane Eduardo (2014). They found that the 
propagation of convection into the upshear regions is the major distinction between intensifying members versus non-intensifying (NI) members, where this feature is not present. The budget analysis of tangential momentum showed that the radial eddy vorticity flux contributed positively (negatively) to the spin up (spin down) process of tangential winds in the middle to upper levels and reduced (increased) vortex tilt in the RI (NI) members. In spin up case, the positive corridors flux term dominates leading to an upright vortex alignment. Once the vortex is aligned, the radial advection of mean vorticity takes over dominating and continuing intensification. (Leighton et.al 2018).

In the following chapters, the large scale interaction in tropical cyclone Irma and Michael using observations and numerical simulations will be discussed. Chapter 2 discussed the motivation and scientific question that drives this research along with the goal, objectives, and hypothesis. Chapter 3 entails a detailed description of the experimental design and methods behind the research along with modeling and post processing tools used. The results from observations and HWRF simulations on each tropical cyclone are presented in Chapter 4. A summary of the key findings and future work are discussed in Chapter 5. 


\section{CHAPTER 2: RESEARCH QUESTION, OBJECTIVES, AND HYPOTHESIS}

\subsection{Motivation}

The motivation behind studying TCs is due to curiosity as a child during the passage of Hurricane Ivan south of Jamaica in 2004. After experiencing the powerful and destructive nature of a hurricane, I developed a passion for understanding TC's intensity behaviors and its impact on society. This chapter seeks to discuss the scientific questions driving this research, the proposed hypothesis behind the research questions, and the goal and objectives accomplished within the research.

\subsection{Focus of Research}

Research on rapid intensifying sheared TC is relatively a newer area of study within RI research. Due to the little research and the lack of knowledge and understanding on this topic, it is well known that there are unanswered questions about the intensification processes under hostile environments. Therefore, the goal of this study is to understand how and why Hurricane Michael developed as a major hurricane despite hostile environmental conditions. What is the role of eddy vorticity fluxes on TC's intensification process? How does the surrounding environment interact with the TC's inner core vortex?

This research was conducted by looking at two different cases of RI under wind shear. The first was Hurricane Irma which, had an almost axis-symmetric development with little interaction with shear. The next was Hurricane Michael, that was heavily influenced by high winds hear and had an asymmetric development. Hence, the objectives of this work can be summarized as follows: 
1)To better understand how the evolution of two varied large scale environments interact and impact the TCs inner cores in each case.

2)To conduct a comparative momentum budget analysis study using high resolution HWRF simulations.

3)To better understand how eddy radial vorticity affects the intensity changes in the TC's tangential wind momentum budgets.

\subsection{Hypothesis}

Based on the fundamentals of asymmetric rapid intensification studies, observation from flight or aircraft reconnaissance, and existing model simulations, here are the main hypotheses of this thesis. The following hypothesis statements are based on the RI in Hurricane's Irma and Michael from a large scale perspective.

\subsubsection{Rapid Intensification of Irma (August 30th/31st)}

Late on August 30th through early August 31st, TS Irma rapidly intensified into a Category 2 hurricane. Favorable conditions prevailed allowing for further development of deep convection and intensification. I hypothesized that favorable conditions and the lack of winds hear or little wind shear allowed Hurricane Irma to rapidly intensify. The spinup process of Hurricane Irma may have occurred in the hurricane boundary layer and eyewall region, where the mean transport of radial influx of absolute vorticity dominates the tangential wind momentum budget.

\subsubsection{Rapid Intensification of Michael (October 8th)}

On October 8th, Michael rapidly intensified from TS to a Category 2 hurricane. Intensification continued despite moderate-to-strong southwesterly wind shear caused by 
an upper level trough over the Gulf of Mexico. I hypothesized that strong convective bursts along with development positive eddy fluxes in the upper troposphere may contribute to Michael rapid intensification in a hostile atmosphere. The positive eddy fluxes may allow for an upper level vortex spin-up by aligning an initial tilt vortex and accelerating the TC spin up process in a top-bottom fashion. 


\section{CHAPTER 3: CASE DESCRIPTIONS, EXPERIMENTAL DESIGN AND METHOD}

\subsection{Case Overview: Hurricane Irma}

Irma, a long-lived TC, started off as a tropical wave with widespread deep convection that departed off the west coast of Africa on August 27th. Irma later became a tropical depression on August 30th 00z located at $16.1 \mathrm{~N}$ and $26.9 \mathrm{~W}$, roughly 120 nautical miles west-southwest of the Cabo Verde Islands. Six hours later, Irma strengthened to a tropical storm (TS) and maintained a westward trajectory south of a midlevel ridge over the Atlantic Ocean. Irma quickly reached hurricane strength after conditions became favorable for development on August 31 06z. Irma made a west-northwest trajectory after a ridge to the north slightly weakened. On September 1 st at 00z, Irma continued to intensify into a major Category 3 hurricane. From September 1 st $00 \mathrm{z}$ to September 4 00z, the intensification process halted after intrusion of dry air and eyewall replacement cycles (ERCs) disrupted the intensification process and caused fluctuations in intensity. Irma changed direction to a west-southwest path after the ridge to the north strengthened, and positioned the TC to move in the direction of high sea surface temperature SSTs (Cangialosi et al. 2018).

Irma reached maximum strength on September 4 after the completion of an ERC and quickly reached $155 \mathrm{kts}(80 \mathrm{~m} / \mathrm{s})$ as a Category 5 . At this point, the TC became better organized with a well-developed eye, and deep convection in the eyewall remained symmetric. On September 6th 06z, Irma made landfall on the island of Barbuda then later in the British Virgin Islands at 16z. Irma continued as a Category $5 \mathrm{TC}$ for more than 48 hours before making landfall in The Bahamas as a Category 4 TC on September 
8. On September 9th, Irma significantly weakened to a Category 2 hurricane after prolonged interaction with land after landfall in Cuba. Irma made a northwest trajectory over the Florida Strait where SSTs were high to support intensification. Irma quickly reintensified into a Category 4 hurricane, 55 nautical miles south-southeast of Key West, Florida. Irma later made a sharp north north-westward turn because it was wedged between an upper level low to the east and a subtropical ridge to the west. On September 10th, Irma made two final landfalls on Cudjoe Key and Marco Island, Florida to where it rapidly decayed due to interaction with the Continental United States. (Cangialosi et al. 2018).

\subsection{Case Overview 2: Hurricane Michael}

Michael, a powerful short-lived TC, originated from a low embedded in a large cyclonic gyre over the northwestern Caribbean Sea. On October 7th 06z, Michael became a tropical depression located 130 nautical miles south of Cozumel, Mexico with a northward trajectory towards the Gulf of Mexico. Michael began a rapid intensification period despite atmospheric conditions being hostile. Michael soon became a TS then a hurricane on October 8th $12 \mathrm{z}$ in an environment of moderate to strong southwesterly wind shear. Michael encountered an abrupt hiatus in intensification as it passed west of Cuba. This was due to the possibility of dry air intrusion, a cold water eddy, and strong wind shear, where a weak convective eyewall structure was observed. (Bevin II et al. 2019).

By October 9th, Michael continued its RI process and continued into a northnorthwestward trajectory toward the continental United States. By October 10th, Michael 
became a major Category 4 hurricane moving on a northward trajectory. The northward motion was due to being positioned between a midlevel ridge and a midlevel shortwave trough over the Gulf Mexico region. Michael RI continued until landfall as a Category 5 hurricane near Mexico Beach, Florida with maximum winds of 140kts $(72 \mathrm{~m} / \mathrm{s})$ at $1730 \mathrm{z}$ October 10th. After landfall, Michael rapidly decayed due to prolonged interaction over land. By October 11th, Michael transitioned into an extratropical cyclone over North Carolina. (Bevin II et al. 2019).

\subsection{Model Description}

For this study, Hurricane Irma (2017) and Michael (2018) were simulated using the triply nested cloud-resolving 4.0 released version of the operational HWRF system. The HWRF system was developed jointly by the National Oceanographic and Atmospheric Administration's (NOAA), National Weather Service (NWS)/ National Centers for Environmental Prediction (NCEP) and the Hurricane Research Division in the Atlantic Oceanographic and Meteorological Laboratories as a part of the Hurricane Forecast Improvement Project (HFIP) (Gopalakrishnan et al 2011, 2012; Tallapragada et al. 2014; Biswas et al. 2018). The HWRF 4.0 released version uses the Message Passing Interface Princeton Ocean Model-Tropical Cyclone (MPIPOM-TC) and NCEP coupler. The HWRF 4.0 released version is the latest version of the model with updated horizontal resolution to $13.5 \mathrm{~km}$ for the parent domain and 4.5 and $1.5 \mathrm{~km}$ for the moving nest. The previous horizontal resolutions were $18 \mathrm{~km}$ for parent domain and 6 and $2 \mathrm{~km}$ for the moving nest. The released version of HWRF decreased the size of domain 2 and domain 3 to $17.8 \times 17.8$ degrees and $5.9 \times 5.9$ degrees, respectively. The vertical levels were 
increased from 61 to 75 with the model a top at 10hPa for all basins (Biswas et al. 2018). An example of the HWRF $13.5 \mathrm{~km}$ parent domain is of Hurricane Michael in (Figure 1). Other improvements in the latest version include improved scale-aware Global Forecast System (GFS) Simplified Arakawa Schubert (SASAS) cumulus parameterization for deep and shallow convection schemes. The Ferrier Aligo cloud microphysical parameterization for explicit moist physics. The Geophysical Fluid Dynamics Laboratory (GFDL) surface-layer parameterization to account for air-sea interaction over warm water and under high-wind conditions. The Noah Land Surface Model (LSM), the Rapid Radiative Transfer Model for GCMs (RRTMG) radiation scheme, the Global Forecast System (GFS) Hybrid Eddy Diffusivity Mass-Flux (HybridEDMF) Planetary Boundary Layer (PBL) scheme (Biswas et al. 2018). 


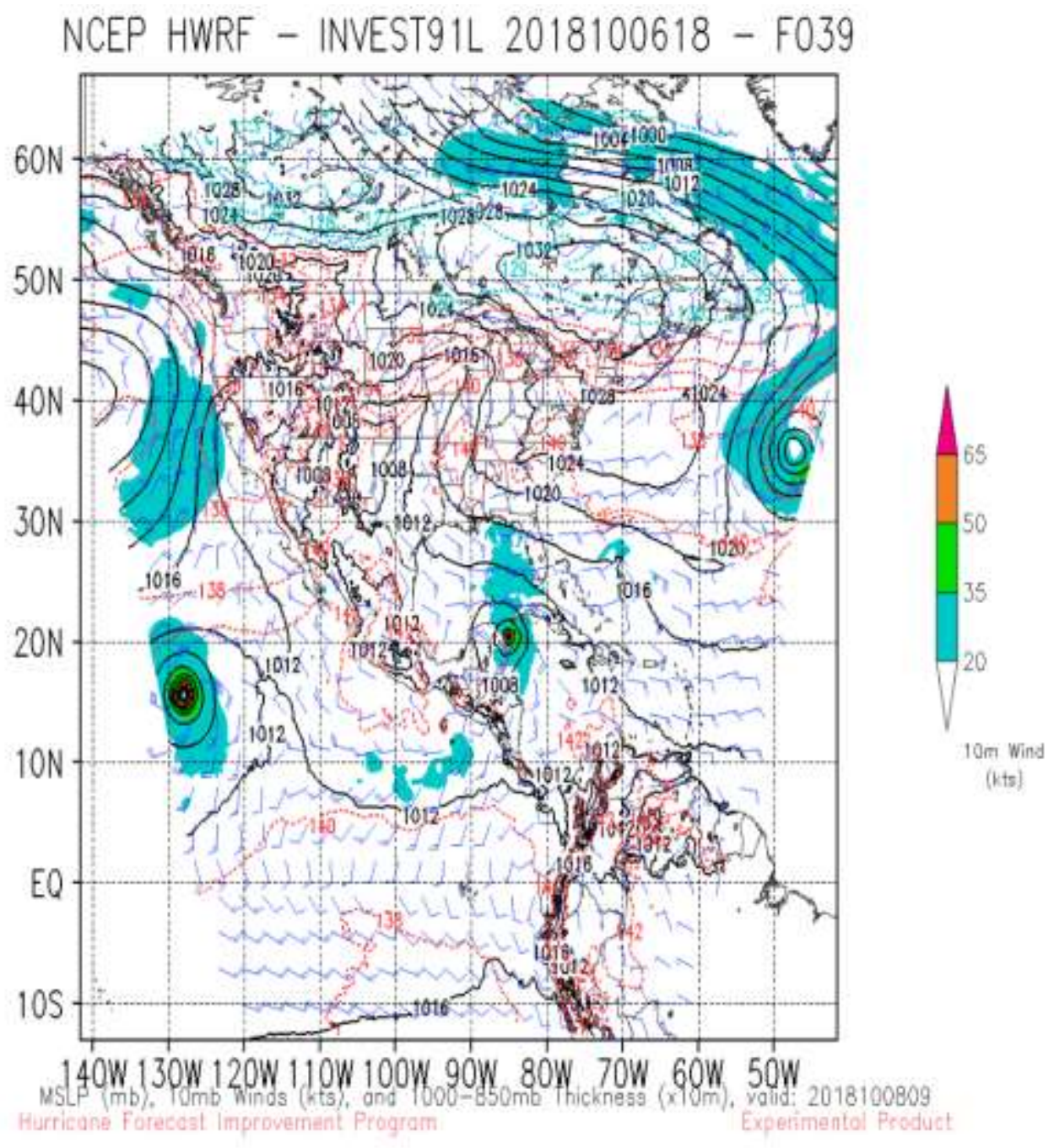

Figure1. Outermost HWRF domain for Michael at 1800 UTC 06 October 2018. (courtesy of EMC)

\subsection{Methods}

The initial conditions and $126 \mathrm{hr}$ boundary conditions for the parent domain are from the GFS forecast. The tangential wind momentum budget equations were added to the WRF-NMM Registry to calculate the budgets of each storm. The diapost was added to the external WRF package io_netcdf component of the WRF interface for post processing of hourly netcdf outputs. The diapost is a diagnostic computing software package 
developed by Drs. Sundararaman Gopalakrishnan, John Michalakes, and Kevin Yeh for post processing of HWRF outputs from netCDF files. This software converts and extracts variables from the HWRF E-grid to an A-grid for analysis. The variables were later used for vertical interpolations of different pressure and height levels to a cylindrical grid. The Grid Analysis and Display System (GrADS) is used for manipulation and visualization of different variables or computation outputs from the diapost processed netCDF files.

Hurricane Irma was initialized at 1200 UTC 30 August 2017 and Michael at 1800 UTC 06 October 2018. The Hurricane Irma case ran for 126hrs and captured cyclogenesis, and the first rapid intensification event during its evolution. The Michael case ran for the same period of time and captured the overall evolution of the storm. This included cyclogenesis, RI, landfall, and rapid weakening. The HWRF large scale environment was compared with the GDAS (GFS) $0.25^{\circ} \times 0.25^{\circ}$ Global Tropospheric Final Analysis to compare environment shear, sea surface temperature, and midlevel moisture against observation. Grb2 outputs were used to plot the large scale features for each case and the diapost was used for diagnostics of the storm vortex and tangential wind momentum budgets.

\subsection{Tangential Wind Momentum Budget Terms}

The approach used in this research is similar to Gopalakrishnan et al. (2019) and Leighton et. al (2018) to better understand the impact of environment shear on the evolution of a TC vortex and to calculate the TC's budgets. We converted the horizontal equations of motion into tangential momentum to a cylindrical polar coordinate system. These terms are divided into sum of means, eddy, and sub-grid scale diffusion (Persing 
et. al 2013; Gopalakrishnan et al 2019). Figure 2 below is the equation for the azimuthally averaged wind tendency used in this study to understand the budgets.

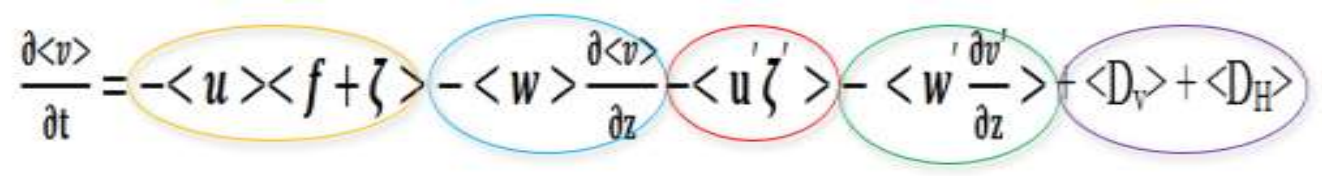

Figure 2. Terms of the tangential momentum budget color coded. Note that $<>$ denotes azimuthal mean and ' (prime) denotes perturbations.

Note that the $<>$ represent the azimuthal average and the prime represents eddy or the departure from the azimuthal mean. The budget terms are colored coded for simplicity in identifying each term. In cylindrical coordinates, $\mathrm{u}, \mathrm{v}$, and $\mathrm{w}$ represents the radial, tangential, and vertical components of velocity. The vertical component of relative vorticity is denoted by $\zeta$ and $\mathrm{f}$ is the Coriolis parameter. The highlighted terms on the right-hand side of the eq. 1 are, the mean radial influx of absolute vertical vorticity (yellow), the mean vertical advection of mean tangential momentum (blue), the eddy radial vorticity flux (red), the vertical eddy advection of eddy tangential momentum (green) and the mean tendency terms due to vertical and horizontal diffusion (purple).

The residual term which includes numerical errors and the pressure gradient were neglected. These errors may originate from interpolation between the model coordinate system to the cylindrical polar system. This is especially valid for this study because the diffusion tendency terms were explicitly computed within the model. We used the outputs from the innermost $(1.5 \mathrm{~km})$ nest for our analysis of the vortex spin-up mechanism in terms of Eq. 1 (Figure 2) in the next chapter. For both cases, the mean storm motion was removed to calculate the tangential wind momentum budget. 


\section{CHAPTER 4: RESULTS}

\subsection{Model Verification (Track and Intensity)}

Figure 3a shows the track for Hurricane Irma against the observed track. Irma's simulated track followed generally close to the observed track with a slightly early shift to the southwest. The simulated track has a slower translation speed than the observed track which could be due to the difference in the surrounding environment. Michael's track (Figure 3b) also follows closely to the observed track. The simulated track, however, has a slight left bias from genesis up to and after landfall.

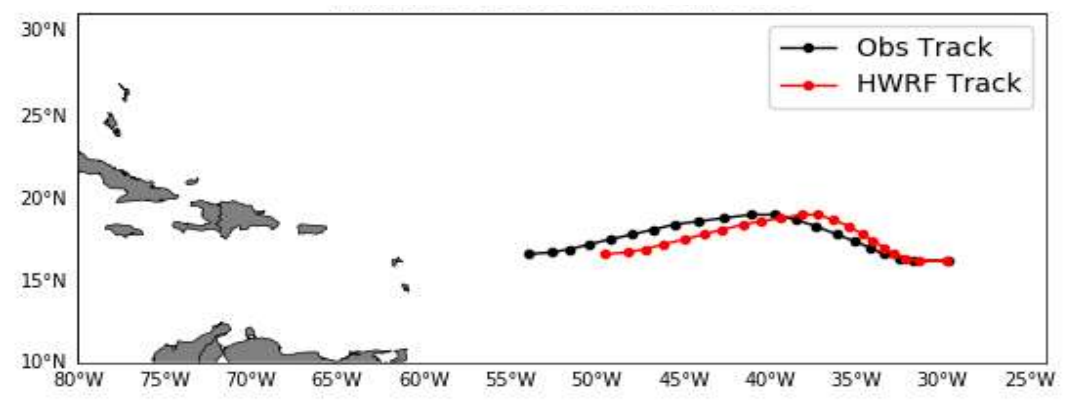

Figure 3a. 5-day forecast track (126hrs) of HWRF Hurricane Irma track (red) initialized at 12 UTC August 30, 2017. The observed track (Best Track) is shown in black. 


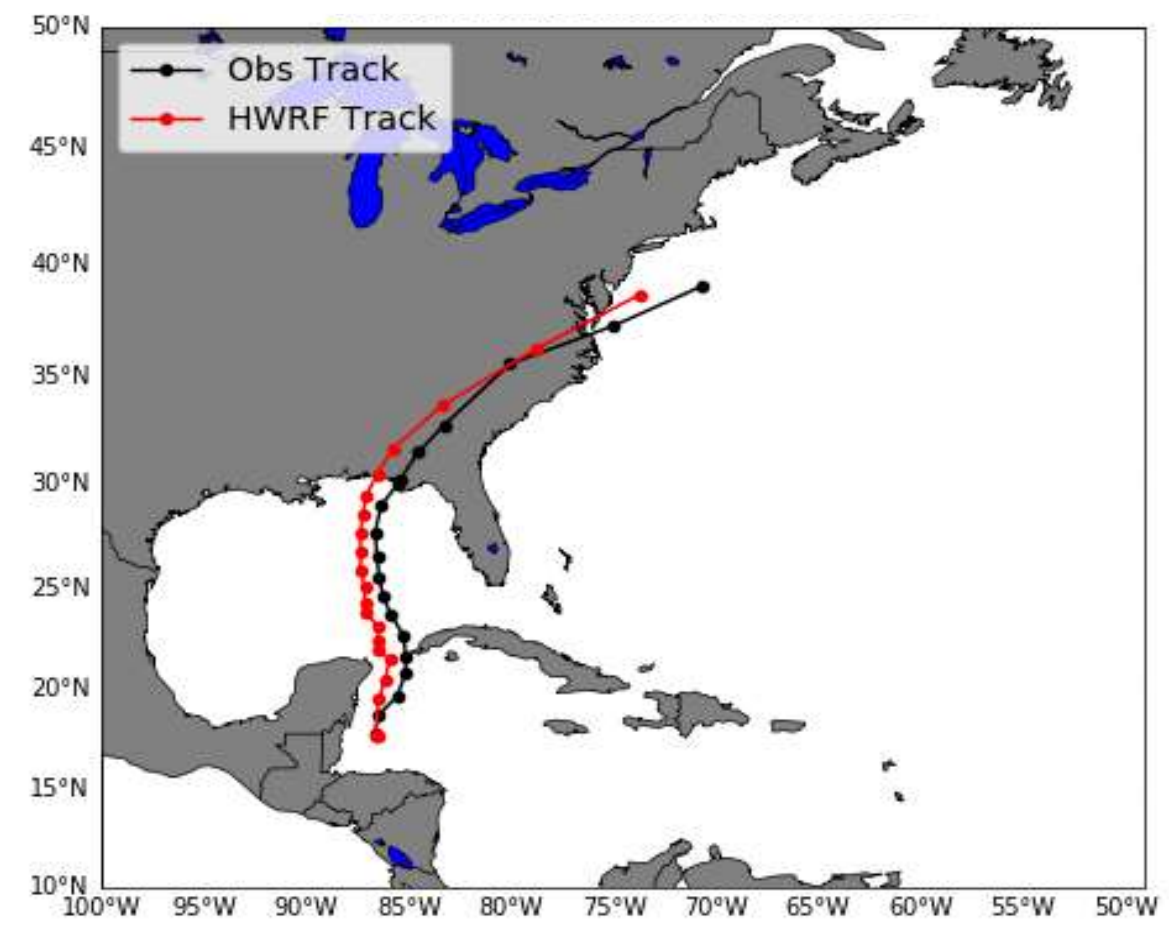

Figure 3b. 5-day forecast track (126hrs) of HWRF Hurricane Michael track (red) initialized at 18 UTC October 06, 2018. The observed track (Best Track) is shown in black.

Figure 4 shows the intensity forecasts for both the minimum central pressure and maximum wind speed for each case. Irma's overall intensity (Figures $4 \mathrm{a}$ and $4 \mathrm{~b}$ ) matched fairly well with the observed intensity. Figure 4a shows the rapid deepening that occurred during the first 24 hours of development. However, the HWRF model had a slightly stronger system throughout the period of RI (0-24hrs), but an overall weaker system after the RI period than the observed. Maximum wind speeds in Figure 4b, are slightly weaker than the observed, with a maximum wind of $52 \mathrm{~m} / \mathrm{s}$ occurring at the 96 th hour. However, 
the model captured the RI period with an increase of $20 \mathrm{~m} / \mathrm{s}$ in wind speed within the first 24 hours.

In contrast, Michael's HWRF forecast experienced disparities in simulated central minimum pressure and maximum wind speeds versus the observed. Figure $4 \mathrm{c}$ and $4 \mathrm{~d}$ shows the evolution of Michael into a weaker system compared to the observed. The forecast had Michael peaked with a minimum pressure and maximum wind speed of 943 $\mathrm{hPa}$ and $57 \mathrm{~m} / \mathrm{s}$ compared to an observed intensity of $919 \mathrm{hPa}$ and $\sim 72 \mathrm{~m} / \mathrm{s}$. A previous study has shown that large disparities in TC intensity prediction (e.g. Zhang and Tao 2013) are due to shear decreasing the predictability of capturing the intensification. Thus, the same can be seen in Michael, where the HWRF model unpredicted the intensity of the system due to the influence of strong wind shear.

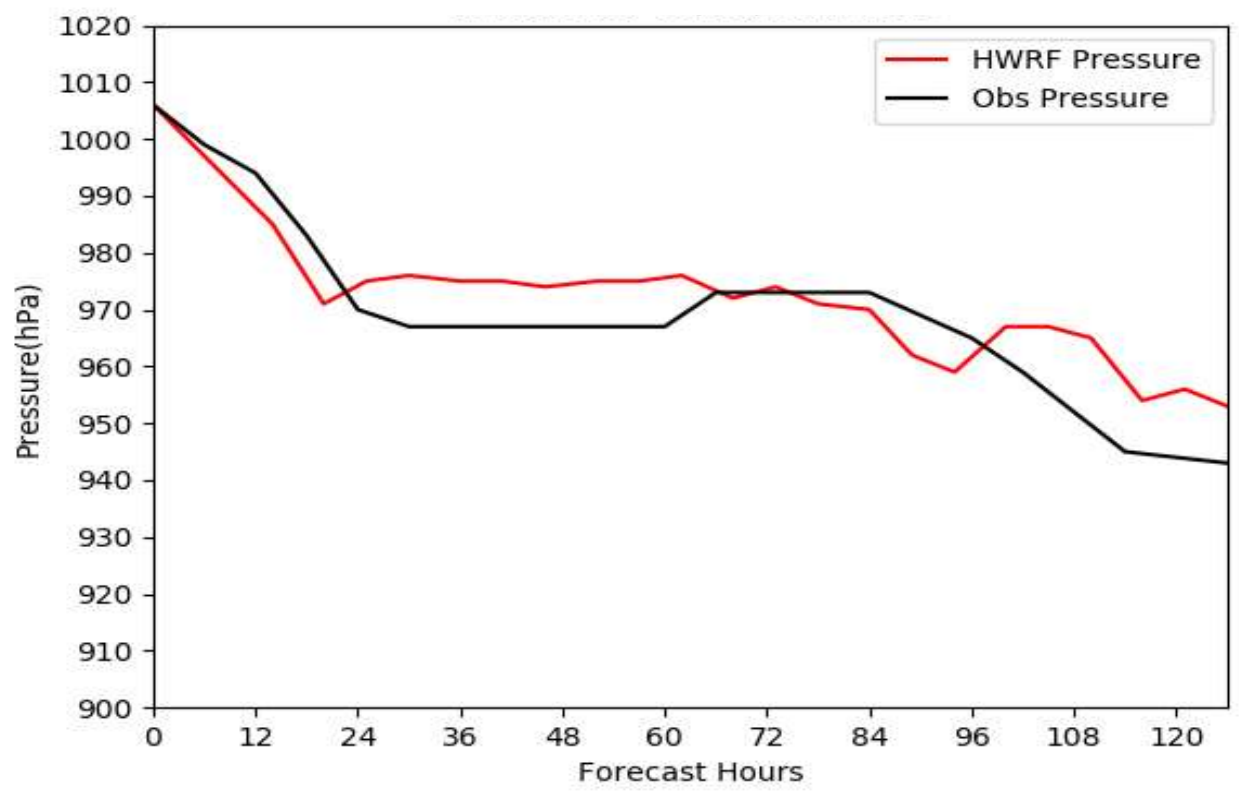

Figure 4a. 126hr Minimum central pressure of Irma HWRF forecast (red) initialized at 12 UTC August 30, 2017 and best track observed pressure in black. 


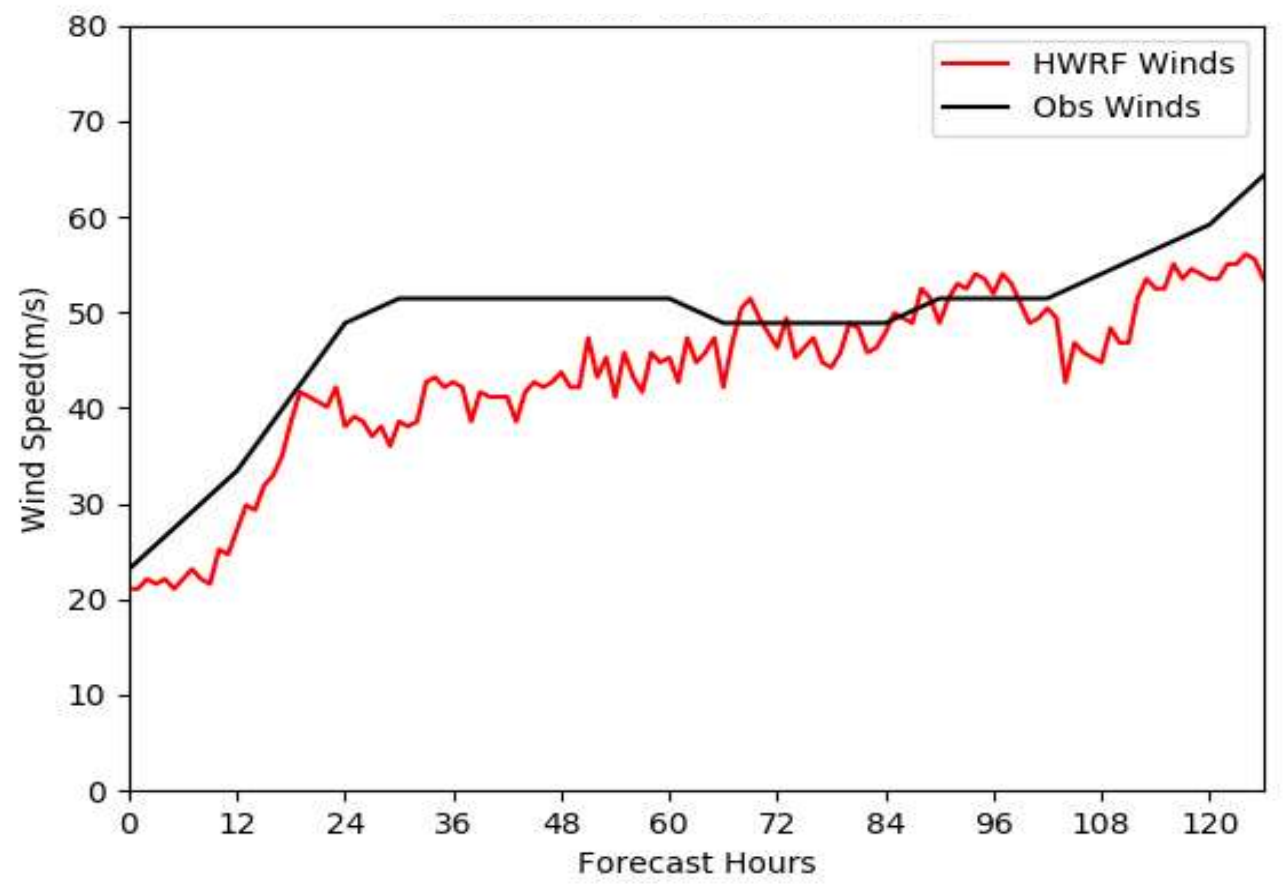

Figure 4b. 126hr Maximum wind speed of Irma HWRF forecast (red) initialized at 12 UTC August 30, 2017 and best track observed pressure in black. 


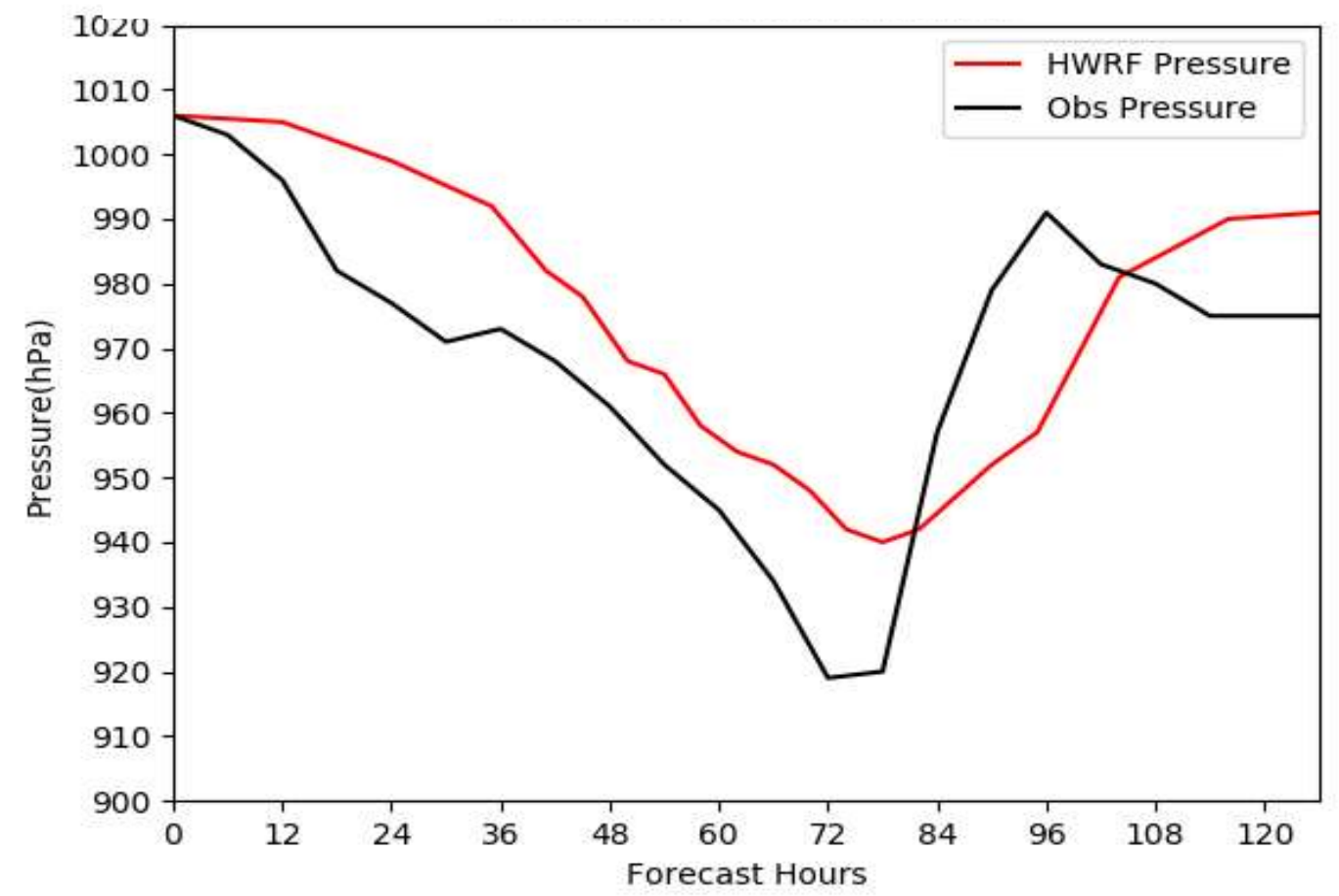

Figure 4c. 126hr Minimum central pressure of Michael HWRF forecast (red) initialized at 18 UTC October 06, 2018 and best track observed pressure in black. 


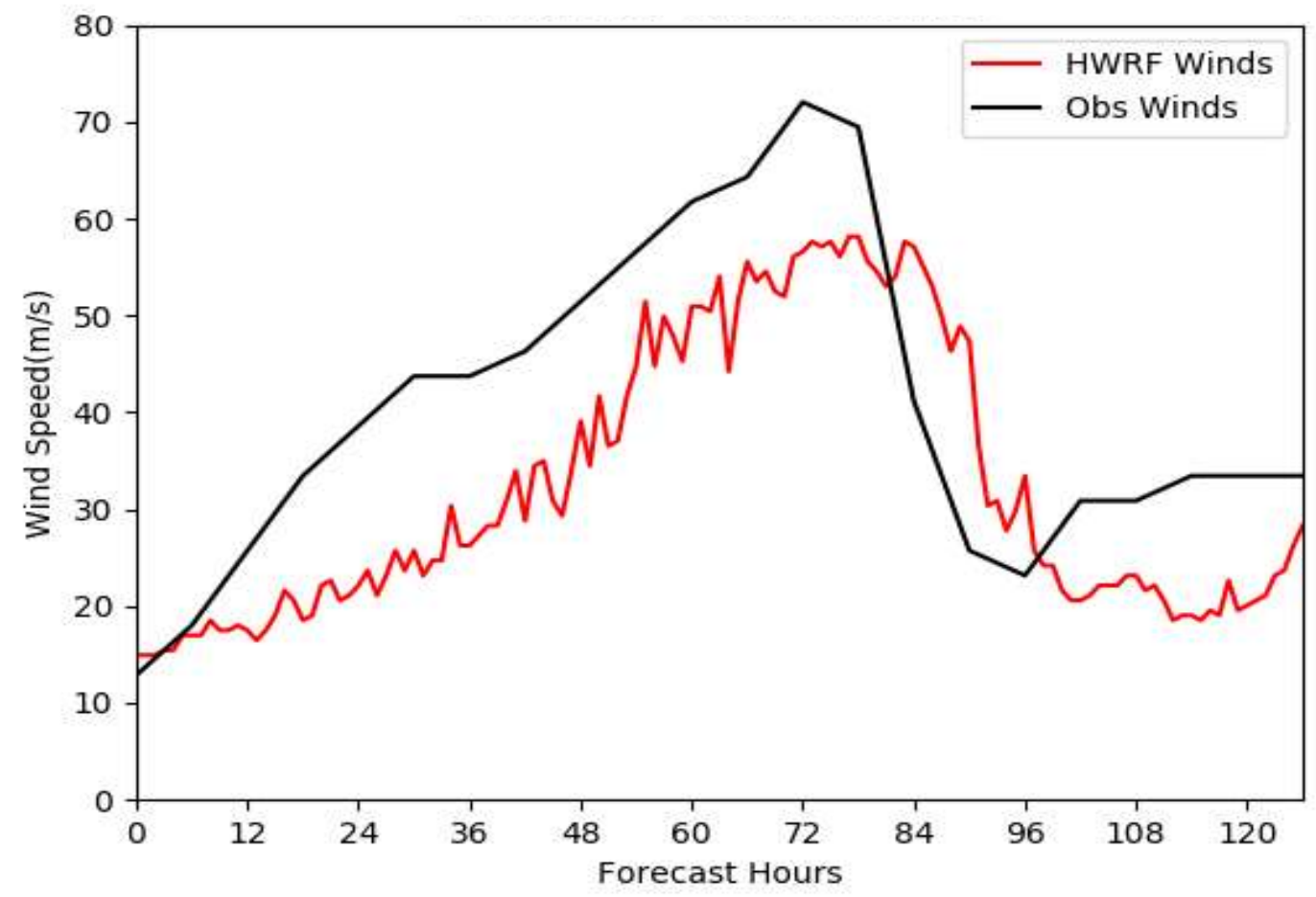

Figure 4d. 126hr Maximum wind speed of Michael HWRF forecast (red) initialized at 18 UTC October 06, 2018 and best track observed pressure in black.

\subsection{Large Scale Wind Shear}

Next, we look at the large scale environment impacts on the intensity changes in each case. Large scale features are compared against the GFS final analysis (observed) to assess the model's potential to reproduce the environment. First, we will look at the Irma case. Figure 5 shows the 850-200 hPa wind shear and $200 \mathrm{hPa}$ streamlines, every 12 hours from 0-24hr. Figures 5a-c correspond and compare to the observed 5d-f respectively. The model did an exceptional job in reproducing the atmosphere compared to the observed. The synoptic upper-level component leading up to, during, after Irma's first RI is as follows. 
Irma started off as a tropical wave that exited the West African Coast with an area of widespread deep unorganized convection. The system moved into an area favorable for development with an atmosphere with very low wind shear. In figures 5 a-c around Irma vortex, the shear magnitude was relatively between $0-5 \mathrm{~m} / \mathrm{s}$. This low sheared atmosphere allows for the system to organize. As Irma moved westward due to the presence of a strong midlevel ridge to its north, the system began to rapidly intensify (Figure 5c). Irma was positioned roughly around $16.4 \mathrm{~N}$ and $32.2 \mathrm{~W}$ when it began its rapid intensification period at 00 UTC August 31, 2017. An upper level trough located to the north-west of Irma with shear magnitudes of $15-20 \mathrm{~m} / \mathrm{s}$ in its right exit region and did not seem to impact the rapid intensification process. Also, there was another shortwave upper trough to the north of Irma had shear magnitude of $15-20 \mathrm{~m} / \mathrm{s}$. Luckily, it was far enough to not have any impacts on shearing Irma's vortex. As the evolution of the atmospheric component changes, Irma continued interacting with a low shear environment, a conducive atmosphere to further intensification. 

A - C
$\mathrm{D}-\mathrm{F}$

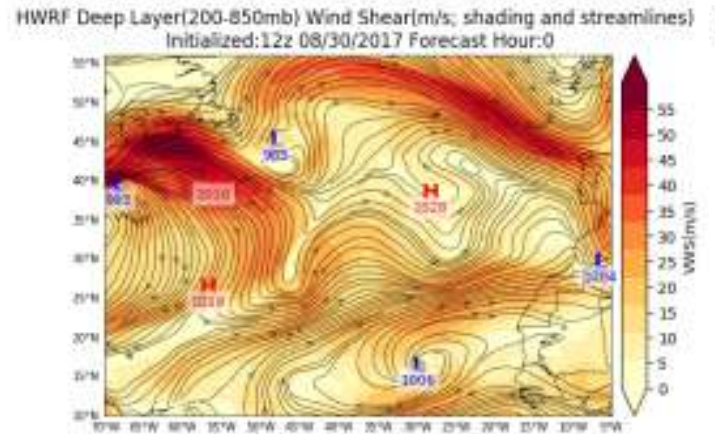

HWRF Deep Layer $200.850 \mathrm{mb}\}$ Wind Shearim/s, shading and streamlines]

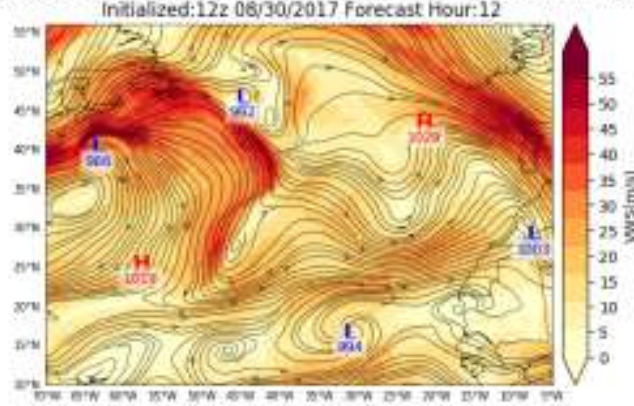

HWRF Deep Layer(200-850mb) Wind Shear(mys; shading and streamlines]

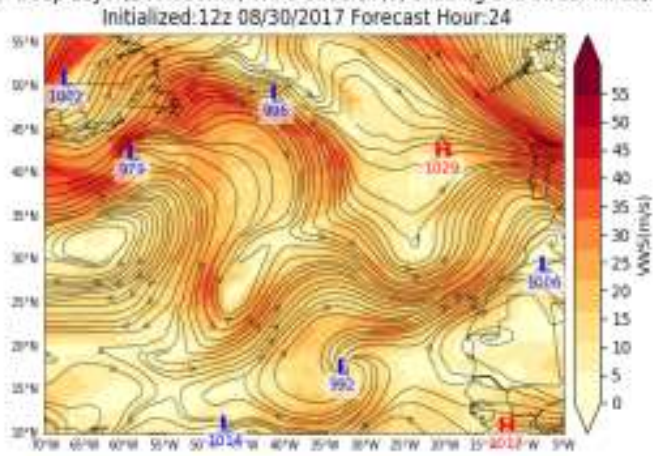

GQAS FNL Deep Loyer(200-850mb) Wird Shearim/s; shading and streamlines) initialized:2017083012

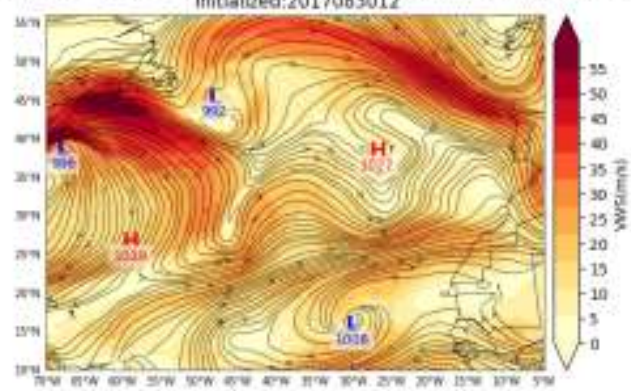

GDAS FNL Deep Layer(200-450mb) Wind Shear(m/s; stading and streamlines) initiallece: 201706310

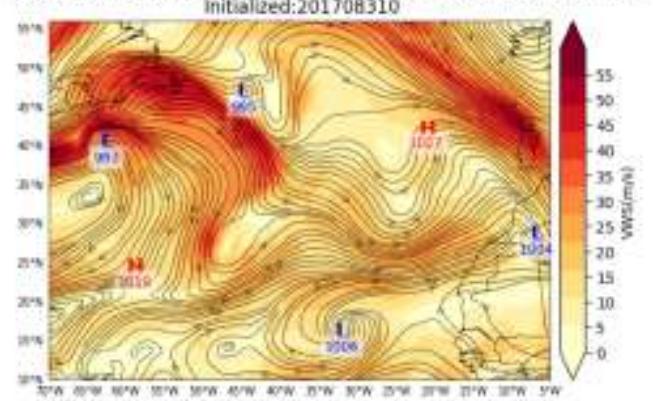

GDAS FNL Deep Layer(200-850mb) Wird Shear(m/s; shading and streamlines) initialized:2017083112

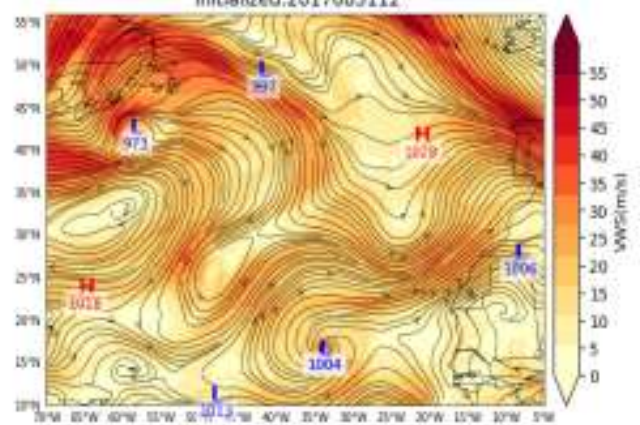

Figure 5. a) 850-200 hPa shear (shaded) and 200-hPa streamlines HWRF Irma forecast at Ohr. b) As in a), but at $12 \mathrm{hr}$. c) As in a), but at 24hr. d) 850-200 hPa shear (shaded) and 200-hPa streamlines GFS Final Analysis at 12 UTC August 30, 2017. e) same as in d), but at 00 UTC August 31, 2017. f) As in d), but at 12 UTC August 31, 2017. Left Column a-c. Right Column d-f. 
Unlike Irma's very conducive atmosphere that supports intensification, Michael's atmospheric component was very complex and hostile, yet RI was achieved. Figure 6 shows the 850-200 hPa wind shear and $200 \mathrm{hPa}$ streamlines, every 12 hours from 2460hr. Figures 6a-d correspond and compare to the observed 6e-h respectively. The model did an exceptional job in reproducing the atmosphere compared to the observed. There are some differences between the model simulation and the observed that I will address. The model simulation produced a stronger upper level trough and a stronger outflow exit region over the Florida Peninsula (Figures 6a-b) compared to the observed (Figure 6e-f) with a weaker out flow. This is one factor that could impact the intensification of the simulation versus the observed.

Michael originated from a large cyclonic gyre over Central America that later reformed its center over the Western Caribbean Sea. Michael developed into a tropical system at 06 UTC October 7, 2018 despite encountering moderate westerly shear from the upper level shortwave trough in the central Gulf of Mexico. As Michael progressed and became better organized, the system began a northward motion around the western branch of the MidAtlantic ridge over the Atlantic (Figures 6a-h). Michael's northward movement increased interaction with the upper level trough to its northwest. Michael began a period of rapid intensification around the 24th hour of the simulation.

Figures 6a and 6e, Michael interacts with the upper level trough in the central Gulf of Mexico with a southwesterly flow around its eastern edge, causing hostile southwesterly shear of $10-15 \mathrm{~m} / \mathrm{s}$. By the 36th hour, the moderate to strong shear continued to impact Michael's vortex structure evolution. However, in Figures $6 \mathrm{~b}$ and $6 \mathrm{f}$, a second upper level 
trough located east of The Bahamas, helped enhance and strengthened the outflow jet region over the Florida Peninsula. Thus, created an area of large upper level diffluence northeast of Michael. From the atmosphere component, this diffluence may have compensated for the hostile shear created by upper level shortwave trough northwest of Michael. The diffluence created an area favorable for the development of deep convection that could resist the impact of wind shear.

Through the 48-60th hour, rapid intensification continued despite the shear. In Figures $6 \mathrm{c}-\mathrm{d}$ and $6 \mathrm{~g}$-h, the upper level shortwave trough weakened and allowed for the shear magnitude to decrease $(\sim 5-10 \mathrm{~m} / \mathrm{s})$. A lower shear magnitude and diffluence to the northeast compensating the shear, could allow for the TC become more vertically stacked and for rapid intensification to commence. Beyond the 60th hour, the outflow to the northeast continued while outflow to the north increased due to the upper level shortwave trough. This also aided the continuation of intensification of the TC by removing air from the upper levels of the TC. 
A - C

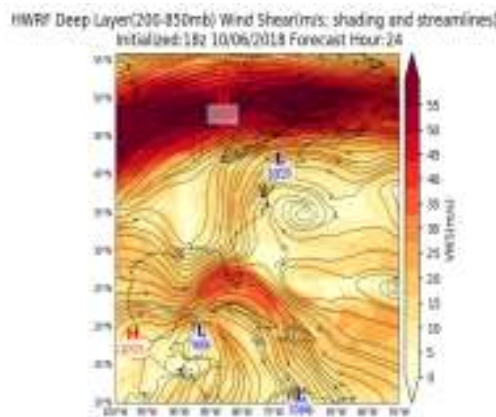

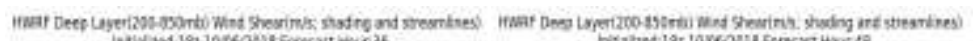
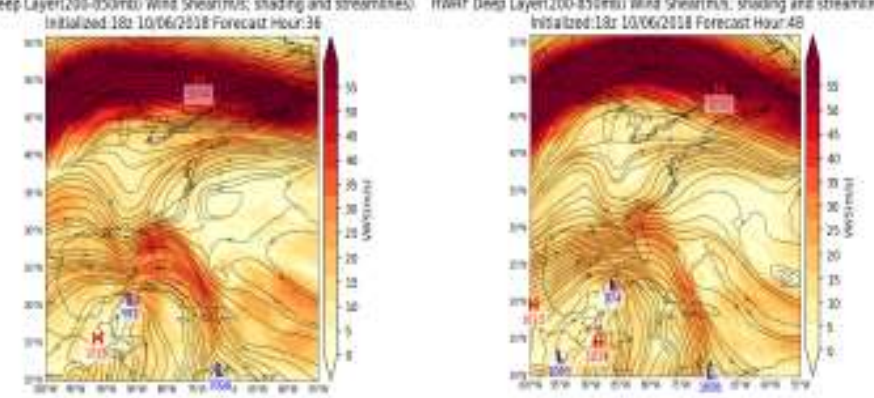

D - F

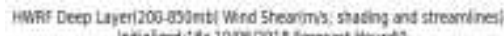

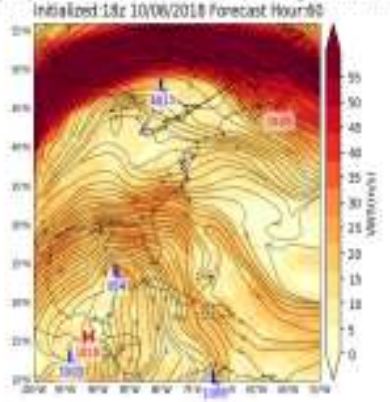

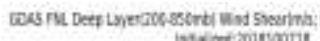

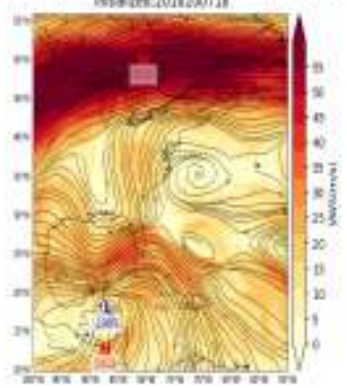

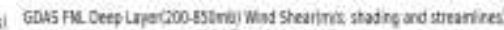
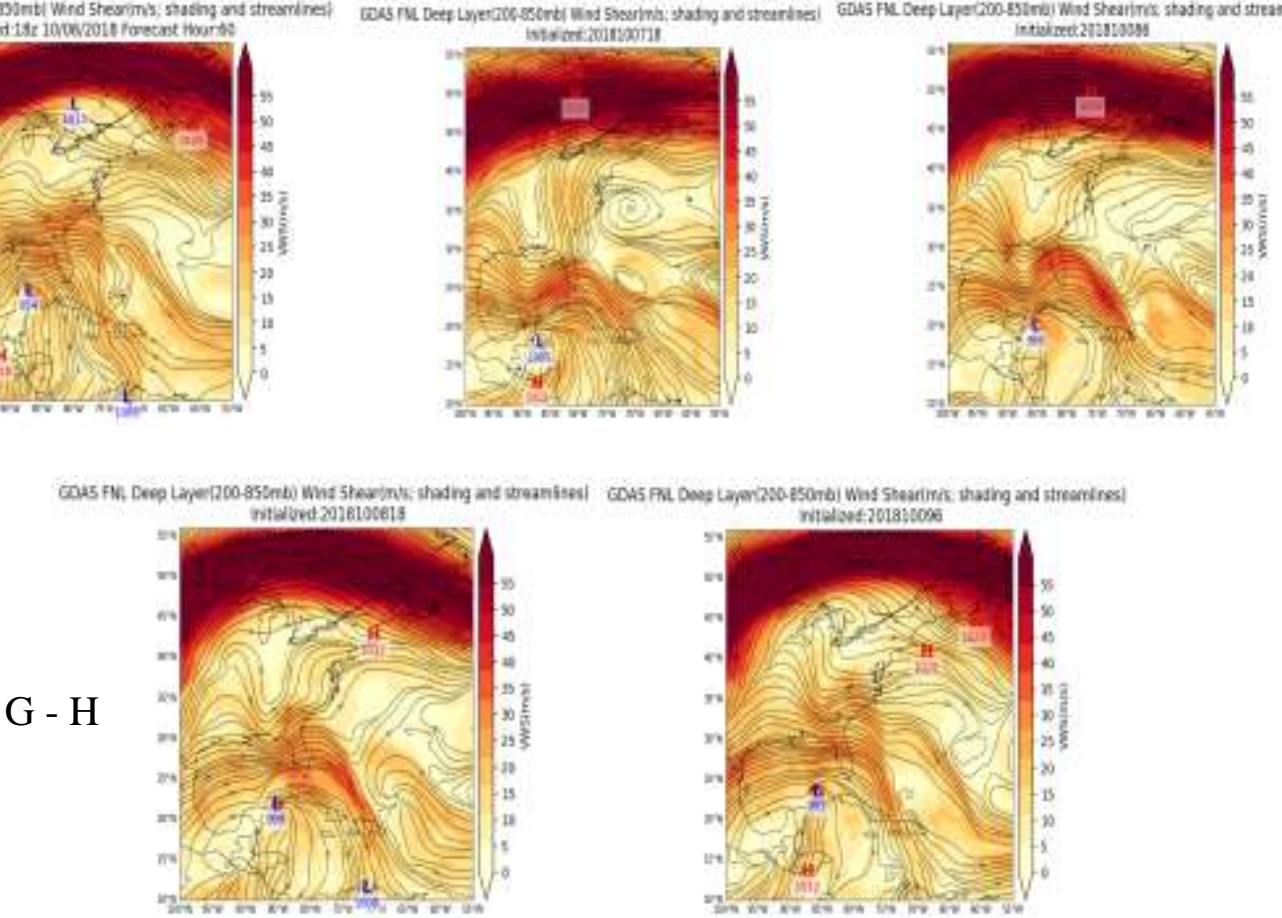

Figure 6. a) 850-200 hPa shear (shaded) and 200-hPa streamlines HWRF Michael forecast at 24hr. b) As in a), but at 36hr. c) As in a), but at 48hr. d) As in a), but at 60hr. e) 850-200 hPa shear (shaded) and 200-hPa streamlines GFS Final Analysis at 18 UTC October 7, 2018. f) same as in e), but at 06 UTC October 8, 2018. g) As in e), but at 18 UTC October 8, 2018. h) As in e), but at 06 UTC October 9, 2018. Left Column a-d. Right Column e-h. 


\subsection{Sea Surface Temperatures}

Next, we look at the SST impacts on the intensity changes in each case. The HWRF simulated SSTs are compared against the GFS final analysis (observed) to assess the model's potential to reproduce the environment. First, we will look at the Irma case. Figure 7 shows SSTs across the Atlantic Basin in the main development region (MDR), every 12 hours from 0-24hr. Figures 7a-c correspond and compare to the observed 7d-f respectively. The model performed generally close in reproducing the ocean temperatures compared to the observed.

A few noticeable differences in the model predictions are seen and are addressed as follows. Noticeable differences can be seen beginning with Figures $7 \mathrm{~b}$ and $7 \mathrm{~d}$. Figure $7 \mathrm{~b}$ has a slightly warm bias compared to the observed Figure 7d. Between $10 \mathrm{~N}, 45 \mathrm{~W}$ and $10 \mathrm{~N}, 55 \mathrm{~W}$, the HWRF model shows warmer SST in that region versus the observed. Just below the TC is also an area of slightly higher SST values of $29^{\circ} \mathrm{C}-29.5^{\circ} \mathrm{C}$ versus the observed $28.5^{\circ} \mathrm{C}$ to $29.5^{\circ} \mathrm{C}$. A slight cold bias is seen northwest of the high pressure symbol in the HWRF results reporting temperatures of $29^{\circ} \mathrm{C}$ versus the observed showing warmer temperatures of $30^{\circ} \mathrm{C}$.

In Figures $7 \mathrm{c}$ and $7 \mathrm{f}$, noticeable differences are seen as well. If you look roughly in the middle of the MDR from $15 \mathrm{~N}, 65 \mathrm{~W}$ to $25 \mathrm{~N}, 65 \mathrm{~W}$ and $15 \mathrm{~N}, 30 \mathrm{~W}$ to $25 \mathrm{~N}$ to $30 \mathrm{~W}$, the HWRF model shows cooler temperatures than the observed. The cold pool of water is shifted slightly more west than observed SSTs. However, below the TC an area of warmer SST can be seen versus the observed temperatures of almost $1^{\circ} \mathrm{C}$ difference. The model result shows SST up to $29.5^{\circ} \mathrm{C}$ whilst the observed shows $28.5^{\circ} \mathrm{C}$. It is important to 
identify these differences to understand how model computation could impact forecast intensity changes.

Now, let us discuss the SSTs impact on Irma intensification through its RI period. At Ohr (Figure 7a), Irma, already a tropical system, was located on the boundary between cooler SSTs to the north and warmer SSTs to the south. The threshold SST value in order for tropical development is roughly $26^{\circ} \mathrm{C}$ (Palmén 1948; Gray 1968; Dengler 1997; Dare and McBride 2011). At SSTs around $27.5^{\circ} \mathrm{C}$, Irma was in an ocean environment that was favorable for development. With SSTs showing $1.5^{\circ} \mathrm{C}$ above the threshold, the ocean provides a source of energy for further development along with atmospheric conditions being favorable.

At the 12th hour of the simulation (Figure 7b), when RI period commenced for Irma, Irma shifted movement to the west-northwest moving into territories of cooler SSTS. In Figure $7 \mathrm{~b}$, Irma moved into slightly cooler water temperature with temperatures of $27^{\circ} \mathrm{C}$. Moving into cooler temperatures pose the possibility of limiting the availability of heat energy needed to support deep convection necessary to sustain intensification.

At the 24th hour (Figure 7c), Irma continued on its west-northwest direction into cooler SST due to the weakening of the MidAtlantic high pressure to the north of Irma. The TC moved into an even cooler SST of $26^{\circ} \mathrm{C}-26.5^{\circ} \mathrm{C}$. At this stage, Irma completed its first RI cycle and the intensification process halted and plateaued. The intensification period resumed two days later after the high pressure to the north re-strengthened and altered Irma's direction back to the west-southwest and eventually moving into warmer SSTs. During this period, Irma experienced a second RI period. 
A - C

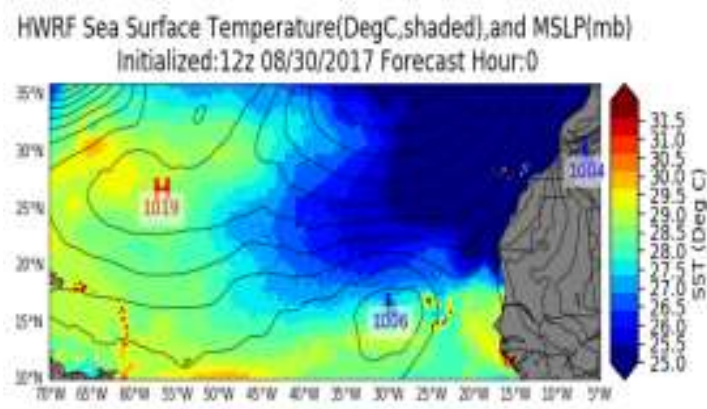

HWRF Sea Surface Temperature(DegC, shaded),and MSLP(mb) Initialized:122 08/30/2017 Forecast Hour:12

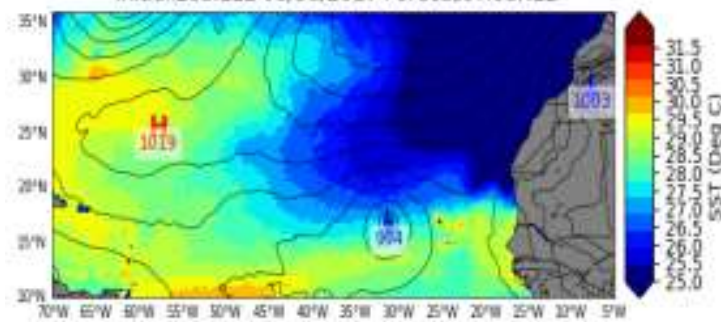

HWRF Sea Surface Temperature(DegC, shaded), and MSLP(mb) Initialized:12z 08/30/2017 Forecast Hour:24

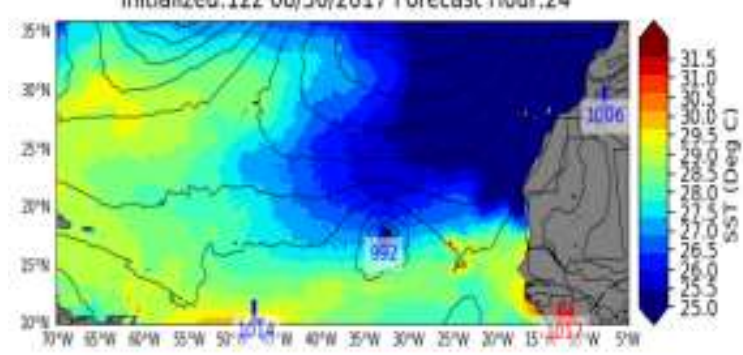

D - F

GDAS FNL Sea Surface Temperature(DegC, shaded), and MSLP(mb) Initialized:2017083012

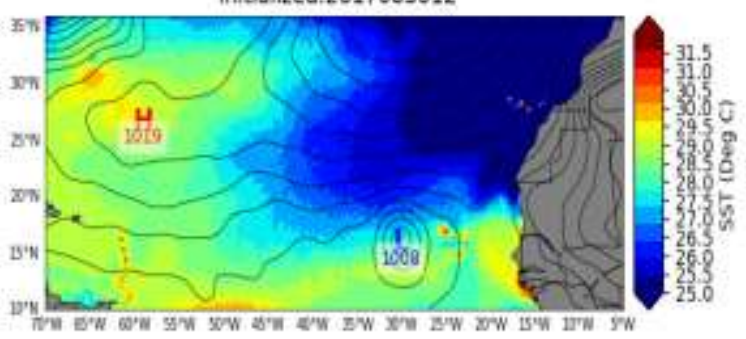

GDAS FNL Sea Surface Temperature(DegC, shaded), and MSLP(mb) Initialized:201708310

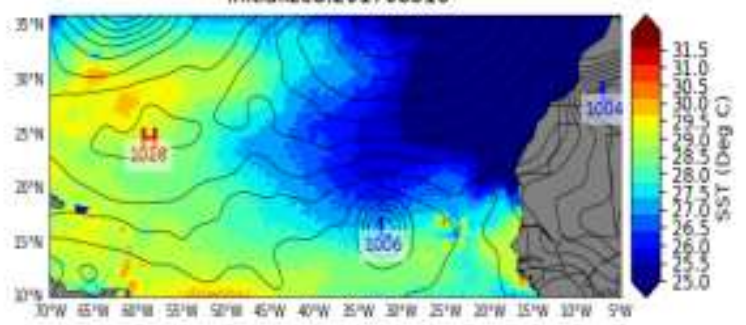

GDAS FNL Sea Surface Temperature(DegC, shaded),and MSLP(mb) Initialized:2017083112

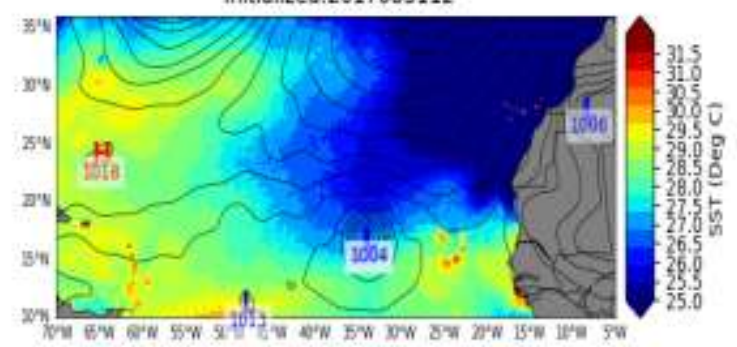

Figure 7. a) Sea Surface Temperatures (shaded) and pressure contours of the HWRF Irma forecast at Ohr. b) As in a), but at $12 \mathrm{hr}$. c) As in a), but at 24hr. d) Sea Surface Temperature (shaded) and pressure contours of the GFS Final Analysis at 12 UTC August 30, 2017. e) same as in d), but at 00 UTC August 31, 2017. f) As in d), but at 12 UTC August 31, 2017. Left Column a-c. Right Column d-f. 
Figure 8 shows the SSTs across the Western Atlantic Basin, every 12 hours from 2460hr. Figures 8a-d correspond and compare to the observed 8e-h respectively. The model did reasonably well in reproducing the SSTs compared to the observed. There are vast differences between the model simulation and the observed starting with Figures 8 a and 8e. The model results show cooler SST values ranging from 0.5 to 1.5 cooler versus the observed.

A similar situation in Figures $8 \mathrm{~b}$ and $8 \mathrm{f}$ illustrates where the northwestern Caribbean Sea and Gulf of Mexico are running cooler than the observed. The Southern Caribbean Sea shows SST values up to 3 degree warmer than the observed temperatures. In Figures 8c-d, cooler SST bias is observed in the Western Gulf of Mexico and Western Caribbean Sea. A warmer bias is present surrounding the waters around Jamaica and a warm bias observed (Figures 8g-h) in the southern Caribbean Sea.

Hurricane Michael was located in an ocean environment conducive for development. During the genesis phase, the SST temperatures were around $28.5^{\circ} \mathrm{C}-29.0^{\circ} \mathrm{C}$. Ocean temperature this warm is able to supply ample amounts of energy to support deep convection and moisten the storm's environment despite shear. The rapid intensification period started around the $24^{\text {th }}$ hour, where the shear magnitude was very hostile for development. At the 24 and 36 hour of the forecast (Figures 8a-b), Michael remained in very warm SST values $3^{\circ} \mathrm{C}$ to $3.5^{\circ} \mathrm{C}$ higher than the threshold. Michael is pulled northward due to an approaching shortwave trough to the north and the Mid-Atlantic High to the east. As seen in Figures 6c-d, SST values remained about the same. At hours 48 and 60 (Figure $8 \mathrm{c}-\mathrm{d}$ ), SST values remained about $28.5^{\circ} \mathrm{C}$ and continued support for 
large ocean fluxes of energy to further intensification. Higher SST values in Michael's case could be one of the main factors that helped compensate against strong shear to support convective development and intensification. 


\section{A - C}
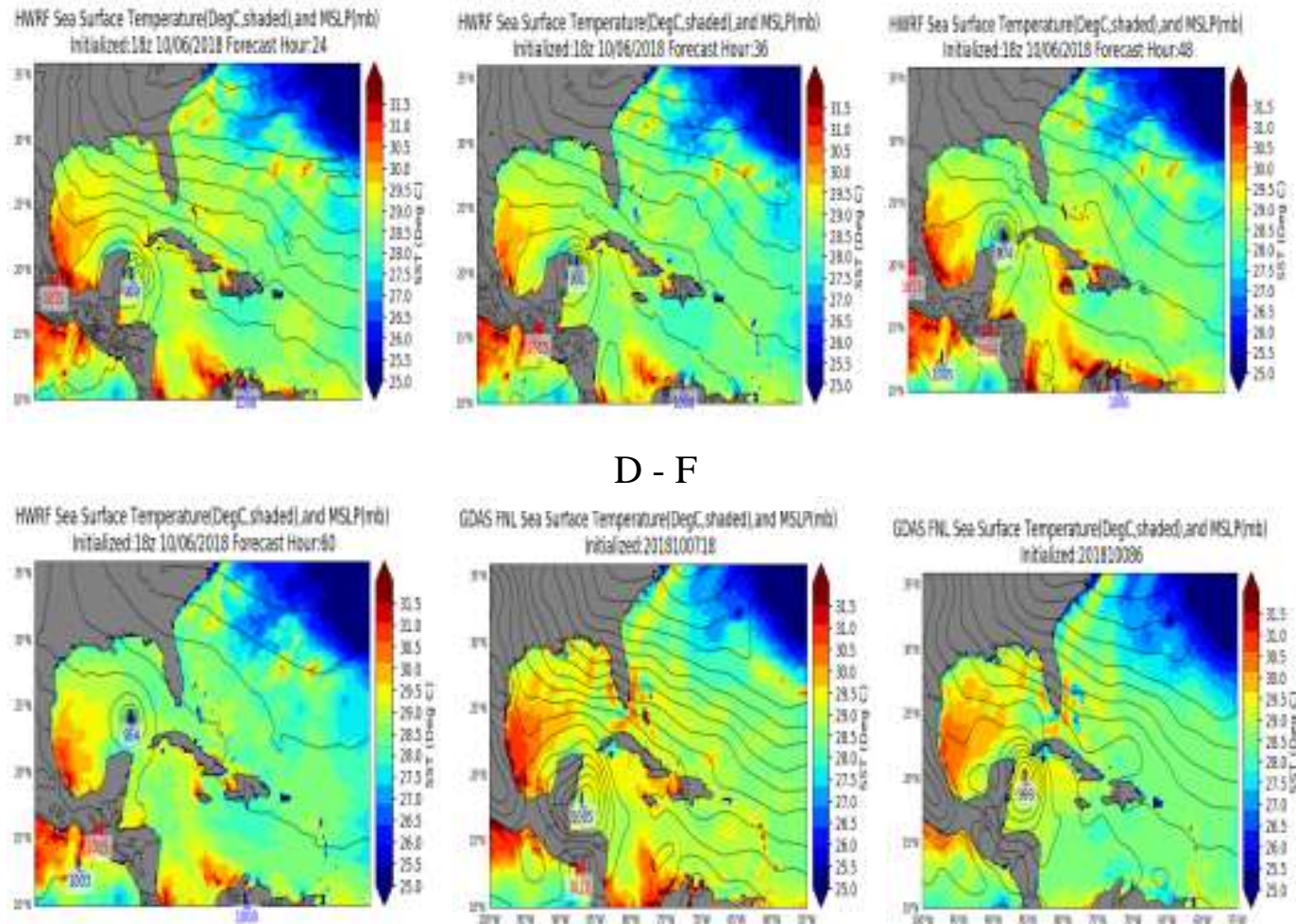

\section{D - F}

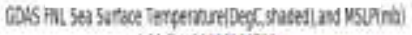
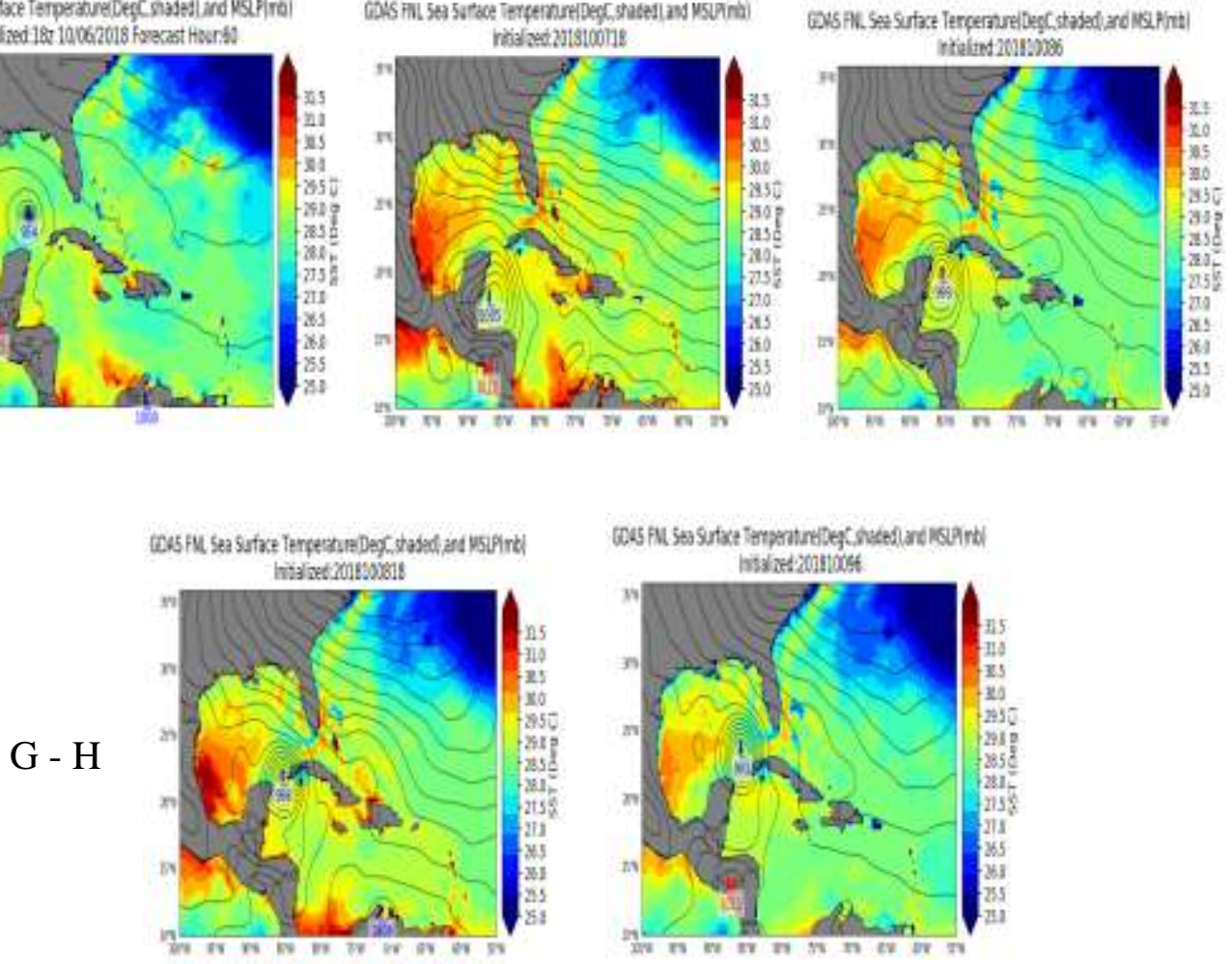

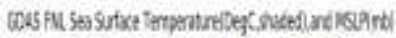

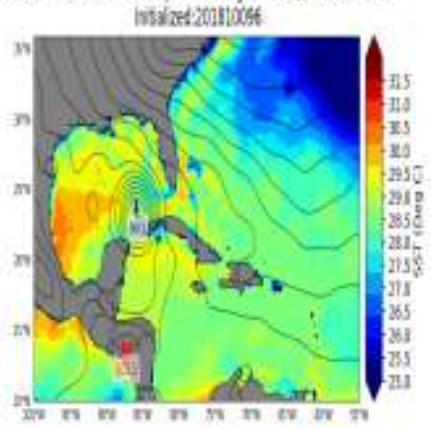

Figure 8. a) Sea Surface Temperatures (shaded) and pressure contours of the HWRF Michael forecast at 24hr. b) As in a), but at 36hr. c) As in a), but at 48hr. d) As in a), but at 60hr. e) Sea Surface Temperature (shaded) and pressure contours of the GFS Final Analysis at 18 UTC October 7, 2018. f) same as in e), but at 06 UTC October 8, 2018. g) As in e), but at 18 UTC October 8, 2018. h) As in e), but at 06 UTC October 9, 2018. Left Column a-d. Right Column e-h. 


\subsection{Midlevel Moisture}

The final large scale component to analyze is the midlevel moisture impact on the structure and intensification process. The HWRF simulated midlevel humidity is compared against the GFS final analysis (observed) to assess the model's potential to reproduce the environment. The midlevel moisture is calculated by taking the weighted average of relative humidity from the $700 \mathrm{hPa}$ to $400 \mathrm{hPa}$ levels. Figure 9 shows relative humidity levels across the Atlantic Basin in the main development region (MDR), every 12 hours from 0-24hr. Figures 9a-c correspond and compare to the observed 9d-f respectively. The model performed well in reproducing the moisture in the midlevels. The forecast shows a slightly moister midlevel environment for Irma (Figures 9a-c) compared to the observed (Figures 9d-f). A big difference in the midlevel moisture can be observed with a wave east of Irma exiting West Africa. The HWRF produced a less robust moist environment compared to the observed.

Studies such as (Kaplan and DeMaria 2003; Hendricks et al. 2010; Wu et al. 2012) have shown that RI of TCs is associated with higher environmental relative humidity in the lower and middle troposphere than non-RI events. Throughout the rapid intensification period, Irma maintained a very moist midlevel environment with relative humidity levels above 80 percent. An environment that moist favors deep convection to support intensification. In Figures 9a-c, a large area of dry air was observed downstream of Irma. The association of drier air in TC's vortex often interrupts the intensification process by limiting the amount of moisture necessary for the genesis and longevity of deep convection and suppressing intensification (Tang and Emanuel 2012; Ge et al. 2013; 
Tao and Zhang 2014). Irma was able to RI despite the presence of drier air to the west and north of the system's environment. These results suggest there is a strong connection between Irma's intensity evolution and the environment. Irma's first RI period occurred in a low shear, relatively moist environment, and over marginal SSTs. 


\section{A - C}

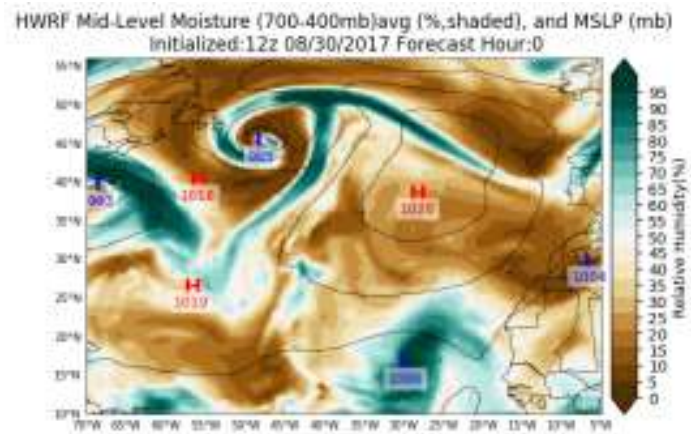

HWRF Mid.Level Moisture (700-400mb)avg [\%, shadedi, and MSLP (mb)

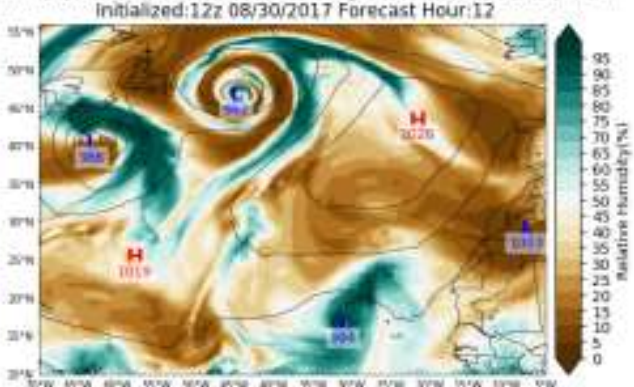

HWRF Mid-Level Mesture $1700-400 \mathrm{mb}$ lavg (\%, shaded), and MSLP (mb)

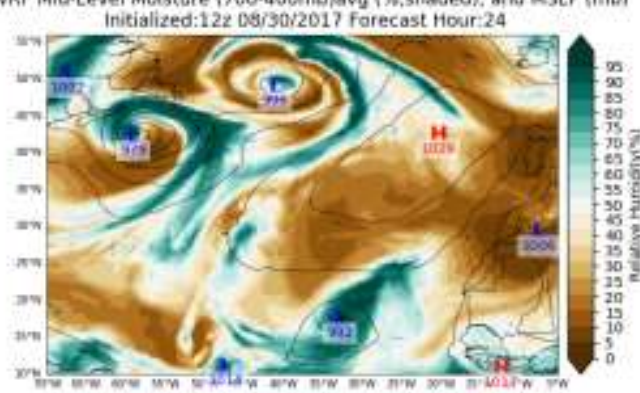

D - F
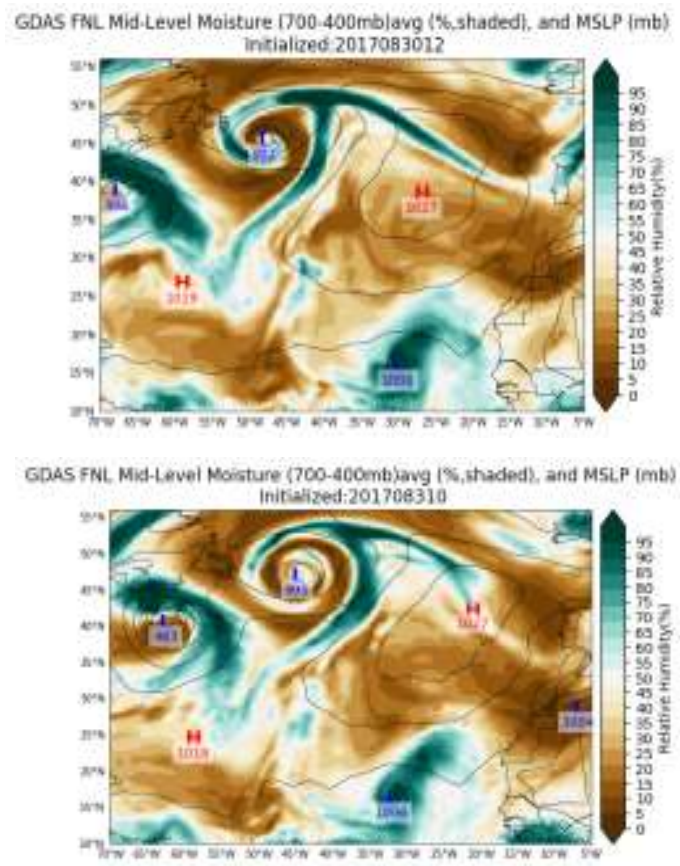

GDAS FNL. Mid-Level Noisture (700-460mblavg Is, shaded), and MSL.P imb)

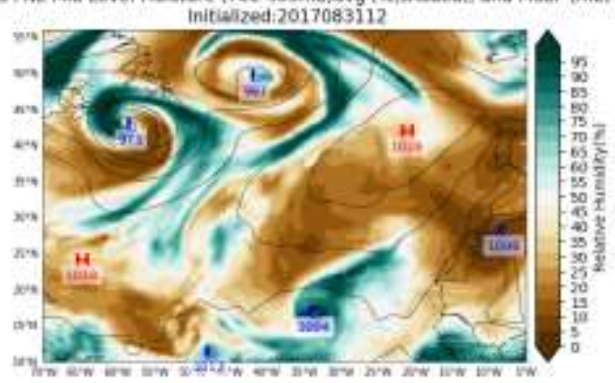

Figure 9. a) Midlevel Moisture (shaded) and pressure contours of the HWRF Irma forecast at $0 \mathrm{hr}$. b) As in a), but at 12hr. c) As in a), but at 24hr. d) Midlevel Moisture (shaded) and pressure contours of the GFS Final Analysis at 12 UTC August 30, 2017. e) same as in d), but at 00 UTC August 31, 2017. f) As in d), but at 12 UTC August 31, 2017. Left Column a-c. Right Column d-f 
Michael's midlevel moisture is more complex compared to Irma's symmetrical moist environment. Figure 10 shows the relative humidity moisture field across the Western Atlantic Basin, every 12 hours from 24-60hr. Like the Irma case, the midlevel moisture is calculated by taking the weighted average of relative humidity from the $700 \mathrm{hPa}$ to 400 $\mathrm{hPa}$ levels. Figures 10a-d corresponded and compared the observed 10e-h respectively. The model did reasonably well in reproducing the relative humidity spread compared to the observed. There are some differences between the model simulation and the observed in all figures. The model results show a more robust impact of dry air intrusion on the western side of the TC compared to the observed.

At the 24th hour of the simulation (Figure 10a), the presence of dry air impacts the TC western boundary limiting the amount of moisture available to produce convection. Moisture levels below 60 percent iseen on the left quadrants of the TC compared to the right with relatively humidity levels 80 percent or higher. This dry air intrusion continued through the RI period where at the 36th hour (Figure 10b), the dry air appeared to penetrate and wrapped on the southern portion of the TC, decreasing the chances for cloud development. At 48th and 60th hour (Figures 10c and 10d), the TC attempts to moisten its environment where a recovery of relative humidity can be seen in the region impacted. Relative humidity levels increased up to 70 percent and is seen on the south and west sides indicating a symmetrization of moisture in the TC environment for convective development and intensification. At this period, the wind shear decreased and SSTs were very warm thus, aided in the recovery of a drier mid- level compared to Irma. 
The presence of dry air and moderate to strong south westerly shear played a major role in altering intensity changes in Michael's evolution. On the other hand, warmer SSTs may have played a significant role in the intensification process by supplying warm humid air up in the midlevels to moisten the environment to support convective development and intensification. 
A - C
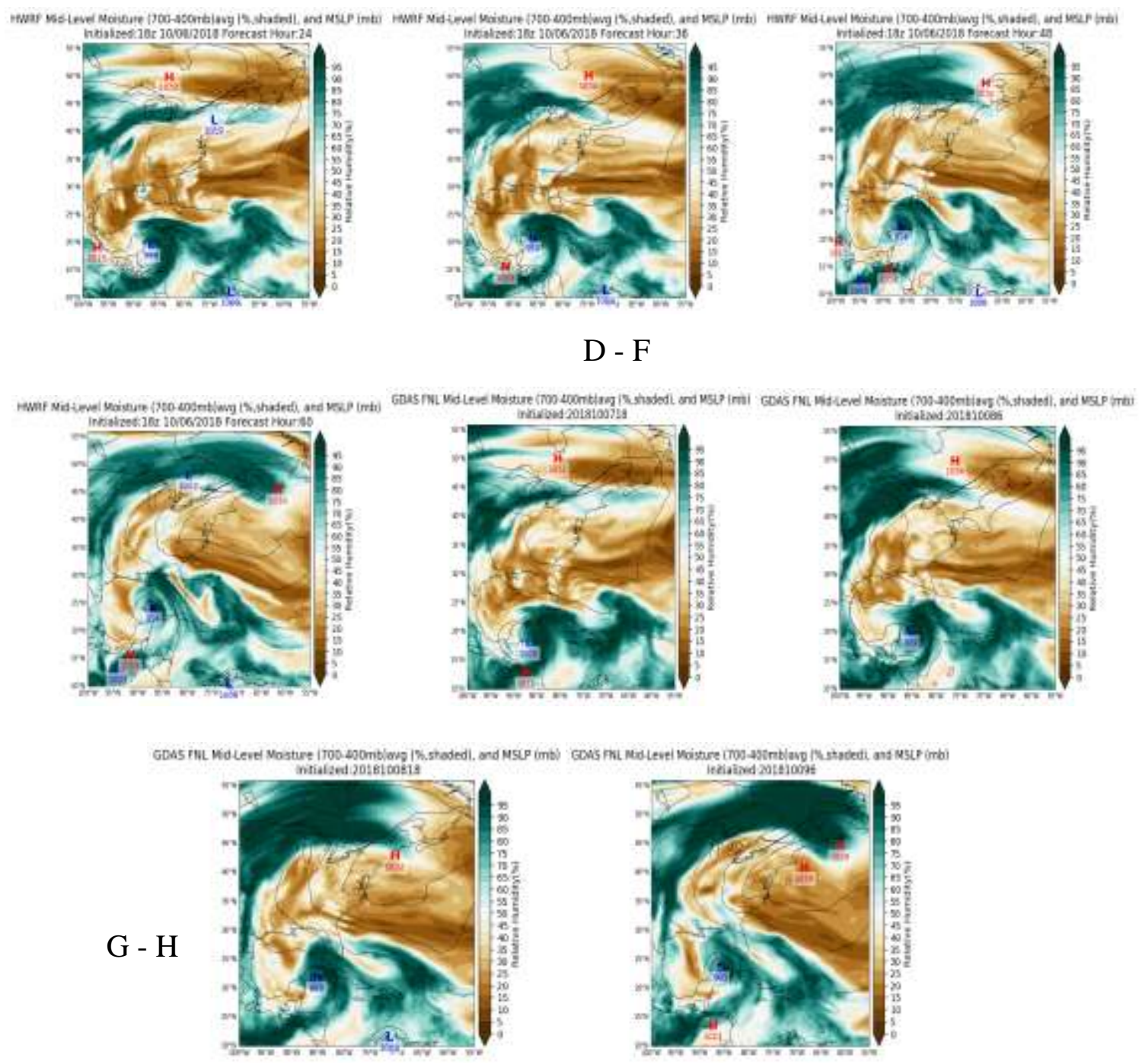

Figure 10. a) Midlevel Moisture (shaded) and pressure contours of the HWRF Michael forecast at 24hr. b) As in a), but at 36hr. c) As in a), but at 48hr. d) As in a), but at 60hr. e) Midlevel Moisture (shaded) and pressure contours of the GFS Final Analysis at 18 UTC October 7, 2018. f) same as in e), but at 06 UTC October 8, 2018. g) As in e), but at 18 UTC October 8, 2018. h) As in e), but at 06 UTC October 9, 2018. Left Column a-d. Right Column e-h. 


\subsection{Inner Core Vortex}

Figure 11 discusses Irma's symmetrical intensification process during the early and late stages of RI. Figures $11 \mathrm{a}$ and $\mathrm{b}$ describes the instantaneous vortex structure of convection and asymmetries associated with TCs in terms of vorticity fields (contours) and vertical velocities (shaded) at the $10 \mathrm{~km}$ height. In all figures, blue shade and contours represent negative values and red shades and contours represent positive values. The black denotes the reference ring of 50km (inner core). During the early stages of RI when the vortex was weak, Figure 11a shows the presence of deep rotating convective plumes in an environment of abundant vorticity around the $12^{\text {th }}$ hour. By the 15 th hour, there are more pronounced convective plumes around the inner core of the vortex. This is consistent with previous studies (e.g. Hendricks et. al 2014; Yang et al. 2007;

Gopalakrishnan et al 2011; Smith 2015). The vortex already took a monopolar structure with positive vorticity suggesting merging and axisymmertization as seen in (Nguyen et al. 2008; and Gopalakrishnan et al. 2019). Irma's vortex was already vertically stacked as a result of the presence of low vertical shear, moist rich midlevel environment, and marginal warm SSTs presented a healthy vortex for continuous strengthening.

During the later stages of RI around the 20th hour (Figure 11b), Irma's vortex structure remains well organized, symmetrical, with rotating deep convection plumes, and an already vertically stacked vortex. Figure $11 \mathrm{~b}$ shows strengthening of vorticity within the radius of maximum winds (RMW). The vortex maintains its monopolar structure with rich cyclonic positive vorticity. In the 20th and 23rd hours, a presence of 
negative vorticity signature of spin down in the center indicating subsidence within the eye. 

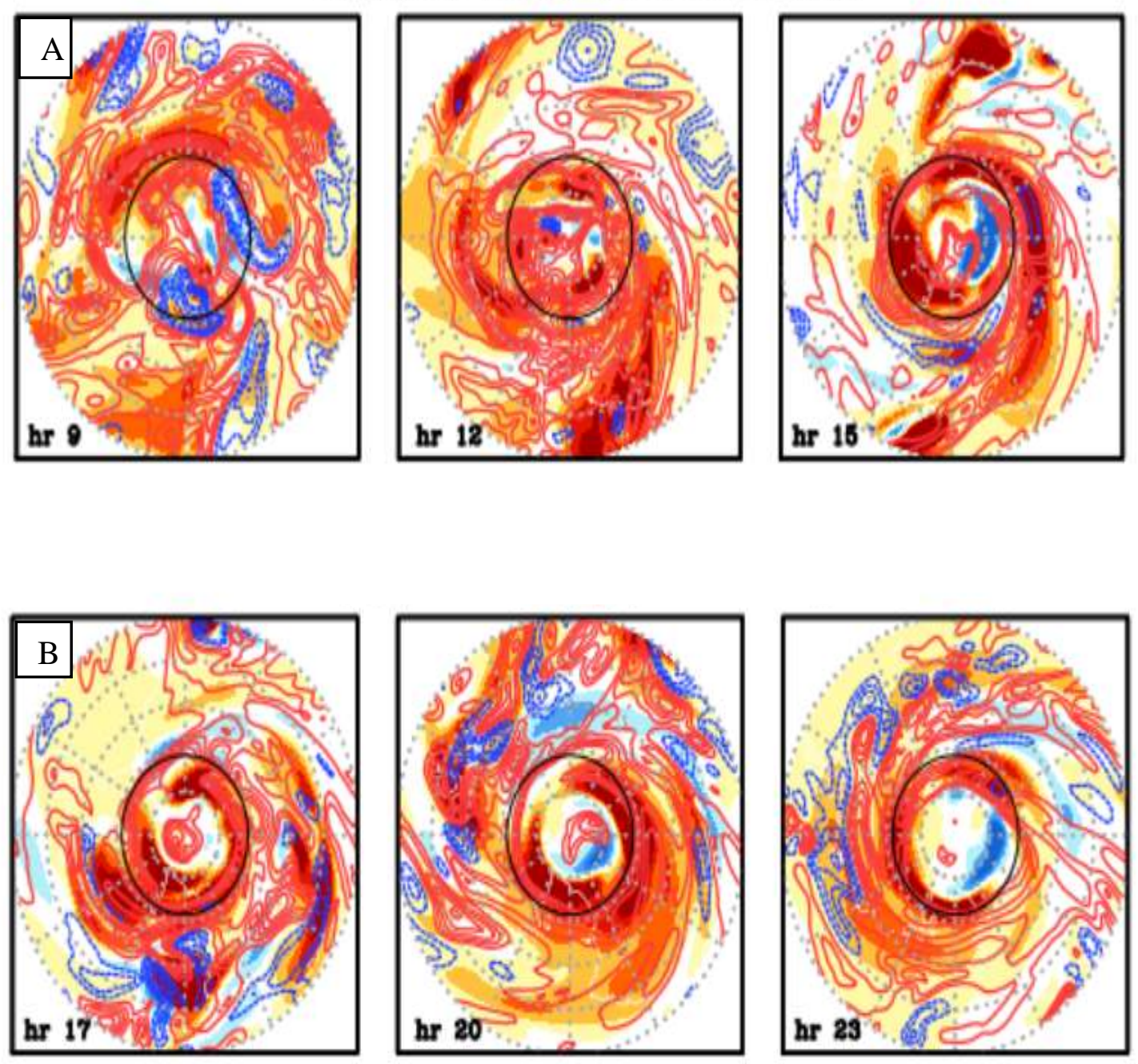

Figure 11. a) (Top) Azimuthal structure of vertical velocity (m/s shaded) with contour vorticity $\left(\mathrm{h}^{\wedge}-1\right)$ at $10 \mathrm{~km}$ height during the RI of Hurricane Irma. b) As in a) (Bottom) but later stage of RI. Note: The black ring indicates the approximate radius of maximum surface winds. Red shades/contours indicate positive values and the blue shades/contours indicate negative values. 
Undoubtedly, Michael's vortex structure takes on a different intensification process than that of Irma. Figure12 shows Michael's asymmetric intensification during the early RI when the vortex was in the titled phase, and later in the aligned phase. Figure $12 \mathrm{a}$ and $\mathrm{b}$ describes the instantaneous vortex structure of convection and asymmetries associated with the TC in terms of vorticity fields (contours) and vertical velocities (shaded) at the $10 \mathrm{~km}$ height. In all figures blue shade and contours represent negative values and red shade and contours represent positive values. The black denotes the reference ring of $50 \mathrm{~km}$ (inner core). The early stage of RI around the 34th hour (Figure 12a), shows a highly asymmetric vortex with convection associated in the downshear left region. This is consistent with findings in studies such as (Chen and Gopalakrishnan 2015; Leighton et al. 2018; Gopalakrishnan et al. 2019). The convective structure of Michael vortex developed from mesoscale convective complexes instead of organized convection plumes in Irma. By the $37^{\text {th }}$ hour, vorticity begins propagation into the upshear shear left region while combating moderate to strong shear.

During the later stages of RI (Figure 12b), shows vorticity intensification with a dipolar (red and blue) pattern at hour 53. At hour 56, there is a wide spread of negative vorticity indicating spin down due to wind shear, yet widespread positive vorticity indicating spin up due to convective activity around the RMW. This system became better organized versus in the early RI states where all the convection was in the down shear left region due to strong south westerly shear. At the $59^{\text {th }}$ hour, vorticity wraps around the center of the system taking on a more symmetrical pattern as Irma. This is an indication that the lower and upper level circulation were better aligned. There is still a 
lot of spin down occurring with the inner core region, but the system continued to intensify. 

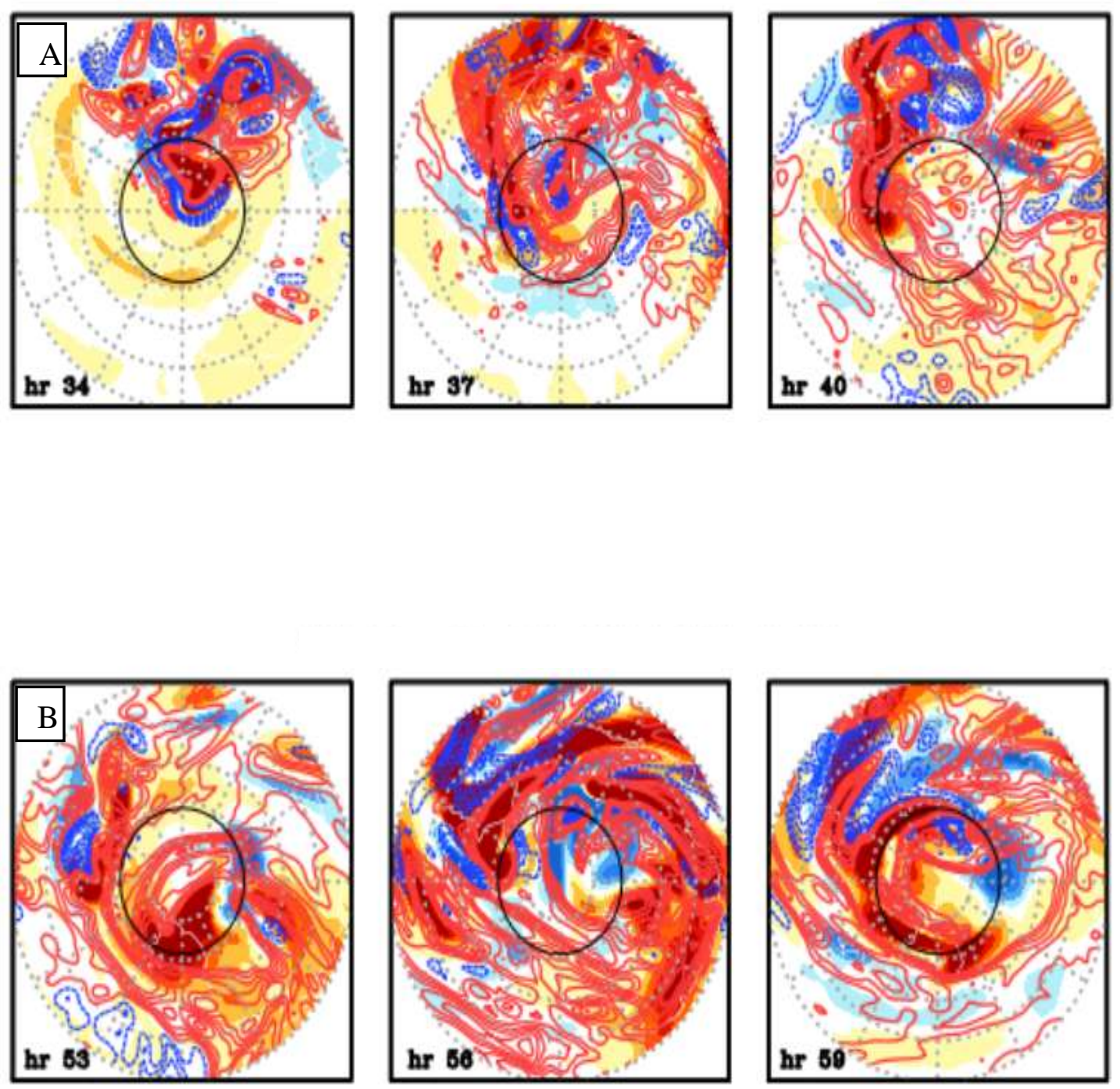

Figure 12. a) (Top) Azimuthal structure of vertical velocity (m/s shaded) with contour vorticity $\left(\mathrm{h}^{\wedge}-1\right)$ at $10 \mathrm{~km}$ height during the RI of Hurricane Michael Titled Phase. $\left.\mathrm{b}\right)$ As in a) (Bottom) but later stage RI in the aligned Phase. Note: Arrow denotes the direction of the shear vector (magnitude of 10-15 m/s) The black ring indicates the approximate radius of maximum surface winds. Red shades/contours indicate positive values and the blue shades/contours indicate negative values. Orange contours indicate maximum values of vertical velocities. 


\subsection{Tangential Wind Momentum Budgets}

Figure 13 identifies various terms in the azimuthally averaged wind tendency equation (eq.1) in Figure 2 for Irma during the early RI phase at the 12th hour. In all figures blue contours represents negative values and red contours denotes positive values of the azimuthally-averaged, 3 hourly time averaged, radius-height cross section of (a) the radial winds $(\mathrm{m} / \mathrm{s}),(b)$ tangential velocity $(\mathrm{m} / \mathrm{s})$, (c) vertical velocity $(\mathrm{m} / \mathrm{s})$. Note the orange contours in (c) represent the maximum values of vertical velocities $(\mathrm{m} / \mathrm{s})$ around the azimuth. (d) Sum of the mean radial influx of absolute vertical vorticity and the mean vertical advection of the mean tangential moment (i.e., the first two terms on the righthand side of the wind tendency equation in chapter 3). (e) The sum of the eddy radial vorticity flux and the vertical eddy advection of eddy tangential momentum and the sum of the vertical and lateral diffusion terms $(\mathrm{m} / \mathrm{s} / \mathrm{h})$. These are the last 4 terms on the right side of the wind tendency equation. (f) The net tendency is the sum of all on the right side of the eq.1 (m/s/h).

During the early stages of RI, the radial circulation is represented by a shallow layer of strong inflow roughly $1 \mathrm{~km}$ deep in the lower troposphere (Figure 13a). A weak inflow layer developed in the midlevels $(5 \mathrm{~km}-9 \mathrm{~km})$ aloft the primary inflow. Above the weak second inflow in the upper level is the presence of a moderate outflow with outflow strengths up to $5 \mathrm{~m} / \mathrm{s}$. Figure $13 \mathrm{~b}$ represents the mean tangential wind associated with Irma from a radius cross-section height view. During this time, Irma is relatively weak with a well developed deep vortex of maximum wind of $25 \mathrm{~m} / \mathrm{s}$ within the hurricane boundary. Figure $13 \mathrm{c}$ shows the mean vertical motions which shows a relatively 
moderate core updraft within the eyewall along with some subsidence within the forming eye.

As for Irma's tangential wind momentum budget, the budget is dominated by the mean transport of radial influx of absolute vorticity and the vertical advection of the mean tangential momentum (Figure 13d). These terms positively contributed to spin up within the eyewall and hurricane boundary region. The eddy terms along with the diffusive terms (Figure 13e) only partially offset the mean transport spin-up. This can be seen in the net (Figure 13e and f) where the radial influx of absolute vorticity is offset by the diffusion term in the lowest level of the hurricane boundary. The spin-down (negative contribution) is prominent in the mid to upper level of the TC which could be influenced by subsidence with the inner eyewall, radial outward advection of vorticity, and eddy and diffusion terms (Figure 13c). The overall net spin up (Figure 13f) in Irma remained positive. This result of Irma is parallel to findings in previous studies that spin up of symmetric TC occurs within the eyewall boundary region (Zhang et al. 2001; Persing et al. 2013; Montgomery and Smith 2014; Gopalakrishnan et al. 2019). There was no positive influence of eddy vorticity flux in the upper levels. 

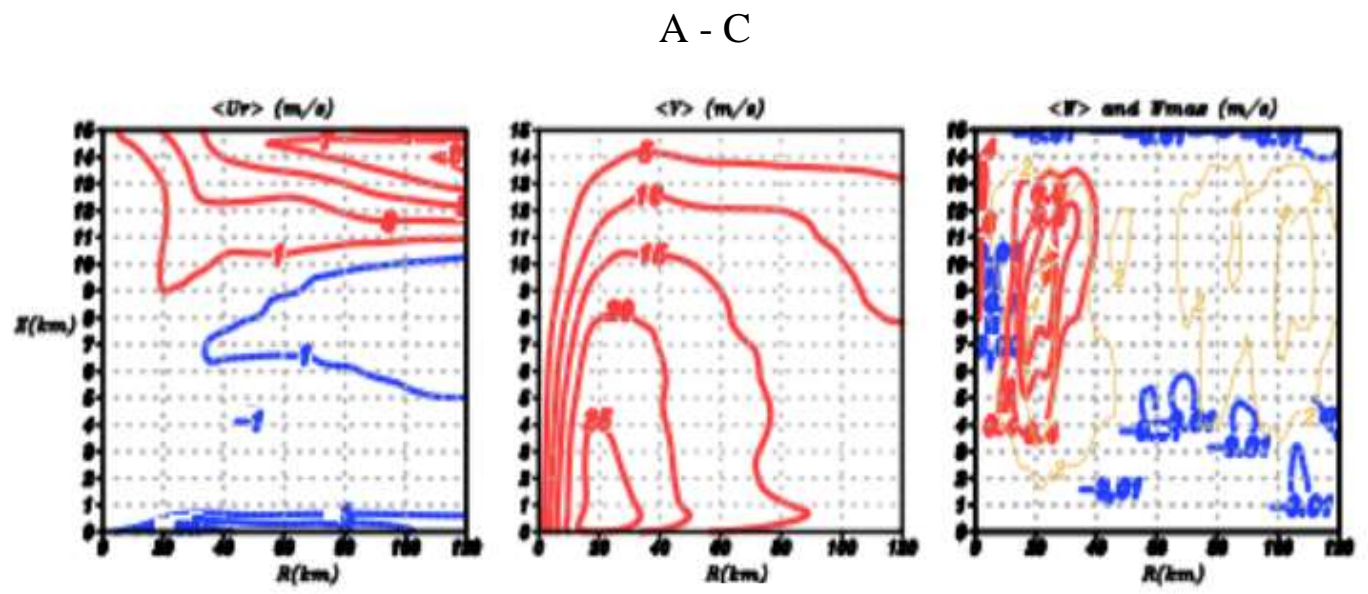

D - E
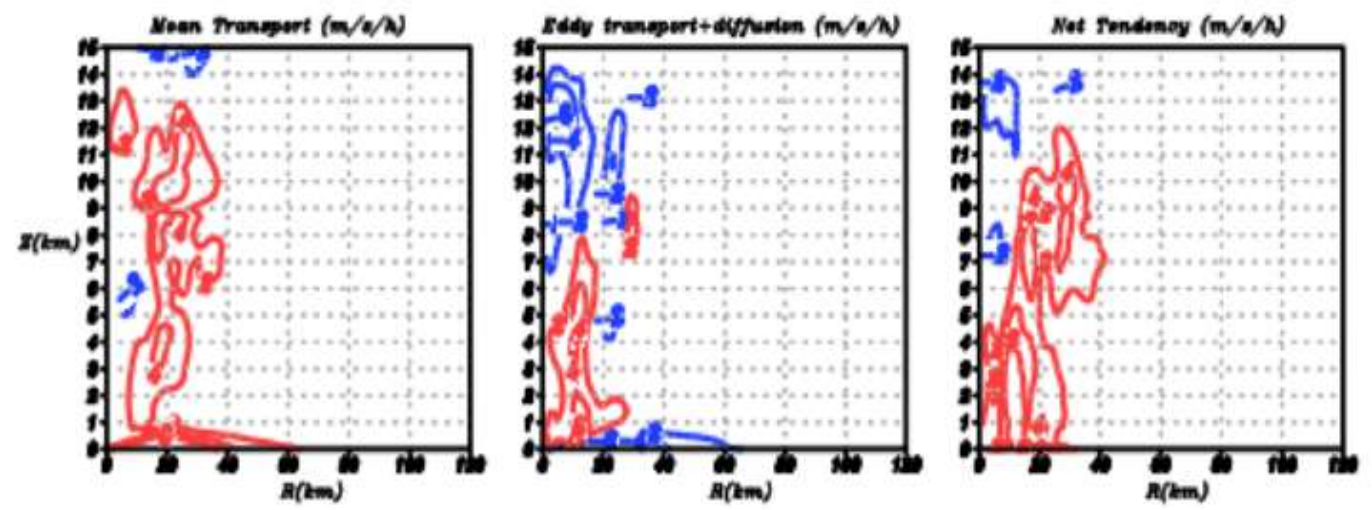

Figure 13. Various terms in the azimuthally averaged tangential wind tendency equation for Hurricane Irma simulation centered at the $12^{\text {th }} \mathrm{hr}$. For all figures, red contours indicate positive values. Blue values indicate negative values azimuthally average, 3 hourly timed averaged, radius height cross-section of a) radial velocity; (m/s), b) tangential velocity $(\mathrm{m} / \mathrm{s})$; c) vertical velocity $(\mathrm{m} / \mathrm{s})$; d) Sum of the mean radial influx of absolute vertical vorticity and the mean vertical advection of the mean tangential momentum $(\mathrm{m} / \mathrm{s} / \mathrm{h}) ; \mathrm{e})$ sum of the eddy radial vorticity flux , the vertical eddy advection of eddy tangential momentum, and sum of the vertical and lateral diffusion terms $(\mathrm{m} / \mathrm{s} / \mathrm{h})$; f) net tendency. Top across a-c. Bottom across d-f. 
During the later stages of RI around the $20^{\text {th }}$ hour, the radial circulation has a strong inflow roughly $1 \mathrm{~km}$ deep in the lower troposphere (Figure 14a). A weak inflow layer remains in the midlevels $(5 \mathrm{~km}-9 \mathrm{~km})$ aloft the primary inflow. Above the weak second inflow in the upper level is the presence of a marginally strong outflow with outflow strengths up to $7+\mathrm{m} / \mathrm{s}$. The mean tangential wind (Figure 14b) grew stronger and a well developed deep vortex passed $16 \mathrm{~km}$ with maximum winds of $40 \mathrm{~m} / \mathrm{s}$ within the hurricane boundary. Figure $14 \mathrm{c}$ shows a strong updraft core on the order of $1 \mathrm{~m} / \mathrm{s}$ within the eyewall along with strong subsidence within the eye down to the lower levels.

As for the tangential wind momentum budget in later stages of RI (Figure 14d), the budget is dominated by the mean transport of radial influx of absolute vorticity and the vertical advection of the mean tangential momentum (positively contributed) within the eyewall and hurricane boundary region (Figure 14a). Again, the eddy and diffusive terms offset the radial flux of absolute vorticity in the lowest level of the hurricane boundary (Figure 14e and f). There is noticeable spin down in the upper levels around the $10 \mathrm{~km}$ $16 \mathrm{~km}$. Spin down in the inner eye wall region is associated with subsidence (Figure 14c). The eddy terms along with the diffusive terms (Figure 14e) contributed to spin down within the entire eyewall region versus just in the upper levels in the early RI stages (Figure 14e). The overall net spin up (Figure 14f) of Irma remained positive due to the mean transport. 
A - C
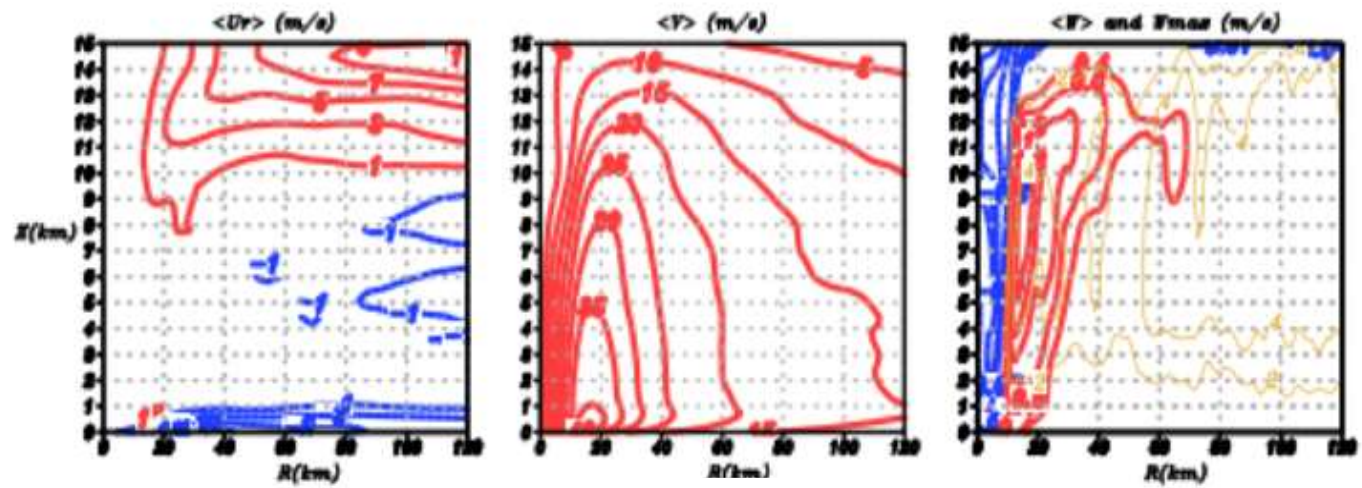

D - E
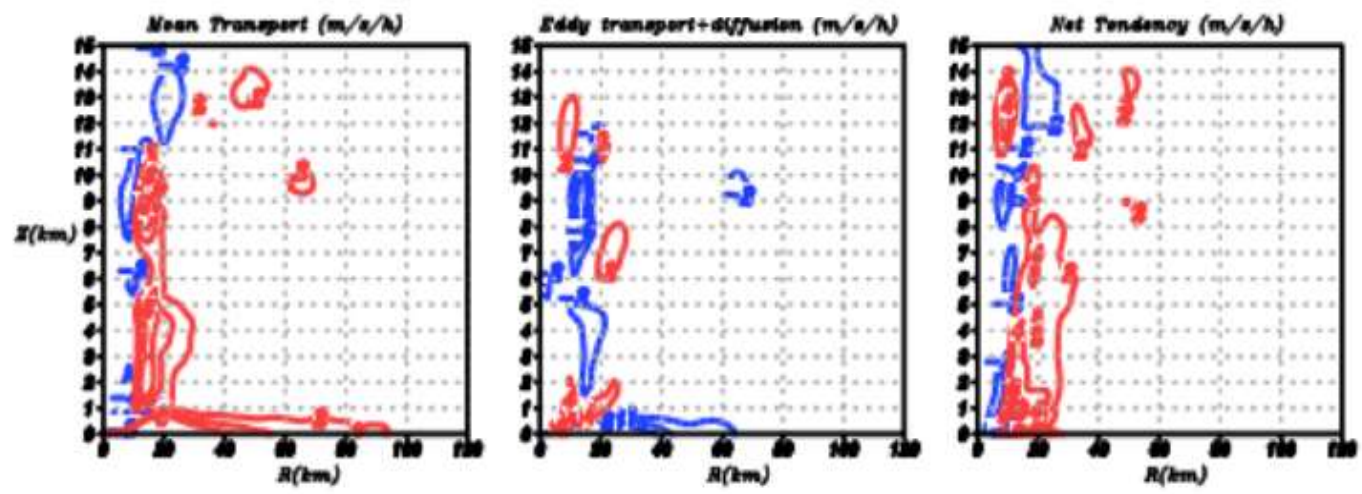

Figure 14. Various terms in the azimuthally averaged tangential wind tendency equation for Hurricane Irma simulation centered at the $20^{\text {th }} \mathrm{hr}$. For all figures, red contours indicate positive values. Blue values indicate negative values azimuthally average, 1 hourly timed averaged, radius height cross-section of a) radial velocity; (m/s), b) tangential velocity $(\mathrm{m} / \mathrm{s})$; c) vertical velocity $(\mathrm{m} / \mathrm{s})$; d) Sum of the mean radial influx of absolute vertical vorticity and the mean vertical advection of the mean tangential momentum $(\mathrm{m} / \mathrm{s} / \mathrm{h}) ; \mathrm{e})$ sum of the eddy radial vorticity flux , the vertical eddy advection of eddy tangential momentum, and sum of the vertical and lateral diffusion terms $(\mathrm{m} / \mathrm{s} / \mathrm{h})$; f) net tendency. Top across a-c. Bottom across d-f. 
Figure 15 identifies various terms in the azimuthally averaged wind tendency equation (eq.1) in Figure 2 for Michael during the early RI phase at the 37 th hour. In all figures blue contours represents negative values and red contours denotes positive values of the azimuthally-averaged, 3 hourly time averaged, radius-height cross section of (a) the radial winds $(\mathrm{m} / \mathrm{s}),(\mathrm{b})$ tangential velocity $(\mathrm{m} / \mathrm{s}),(\mathrm{c})$ vertical velocity $(\mathrm{m} / \mathrm{s})$. Note the orange contours in (c) represent the maximum values of vertical velocities $(\mathrm{m} / \mathrm{s})$ around the azimuth. (d) The sum of the mean radial influx of absolute vertical vorticity and the mean vertical advection of the mean tangential moment (i.e., the first two terms on the right-hand side of the wind tendency equation in chapter 3). (e) The sum of the eddy radial vorticity flux and the vertical eddy advection of eddy tangential momentum and the sum of the vertical and lateral diffusion terms $(\mathrm{m} / \mathrm{s} / \mathrm{h})$. These are the last 4 terms on the right side of the wind tendency equation. (f) The net tendency is the sum of all on the right side of the eq. $1(\mathrm{~m} / \mathrm{s} / \mathrm{h})$.

During the early stages of RI, there was a significant vortex tilt with height. However, the radial circulation was weak with a deep layer of inflow up to $4 \mathrm{~km}$ (Figure 15a). A moderate outflow developed aloft with outflow strengths up to $7 \mathrm{~m} / \mathrm{s}$. Figure $15 \mathrm{~b}$ shows a rather relatively weak mean tangential wind with a developed deep vortex of maximum wind of $20 \mathrm{~m} / \mathrm{s}$ within the hurricane boundary. At this stage, the vortex of Irma (Figure13b) is way more pronounced than that of Michael. Figure 15c represents the mean vertical motions which shows a relatively weak and unorganized core updraft within the eyewall. Compared to Irma, the updraft was consistent through the eyewall 
region (Figure 13c). Figure 15c shows spin up in the upper levels where updraft is present and spin down (downdraft) in the lower levels.

The effects of the downdraft can be seen (Figure 15d), where no spin up occurred in the area where the downdraft is located. Similar to Irma, the budget of Michael is dominated by the mean transport of radial influx of absolute vorticity and the vertical advection of the mean tangential momentum. The mean transport positively contributed to spin up within the eyewall and hurricane boundary region (Figure $15 \mathrm{~d}$ ). However, Irma spin up was consistent throughout the eyewall region where Michael's has no spin up occurring in the midlevels. This could be due to a weaker updraft and the presence of downdrafts in the midlevels of Michael's secondary circulation(15c). The vertical and lateral diffusive terms (Figure 15e) negatively contributed to the spin up process in the hurricane boundary layer. Noticed spin down occurred in the mid to upper levels of the eyewall region could be due to the presence of $10-15 \mathrm{~m} / \mathrm{s}$ of shear. The overall net tendency budget remained positive with spin up occurring up to $120 \mathrm{~km}$ away from the eye. This is not the case with Irma, where spin up solely occurred in the eyewall region. 
A - C
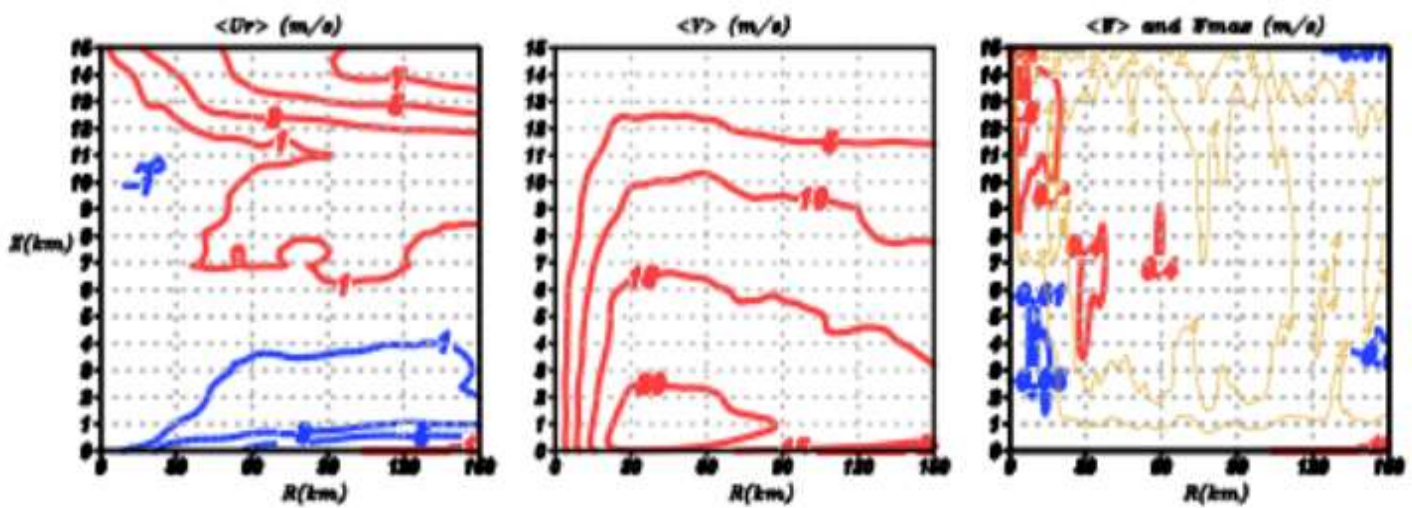

$\mathrm{D}-\mathrm{E}$
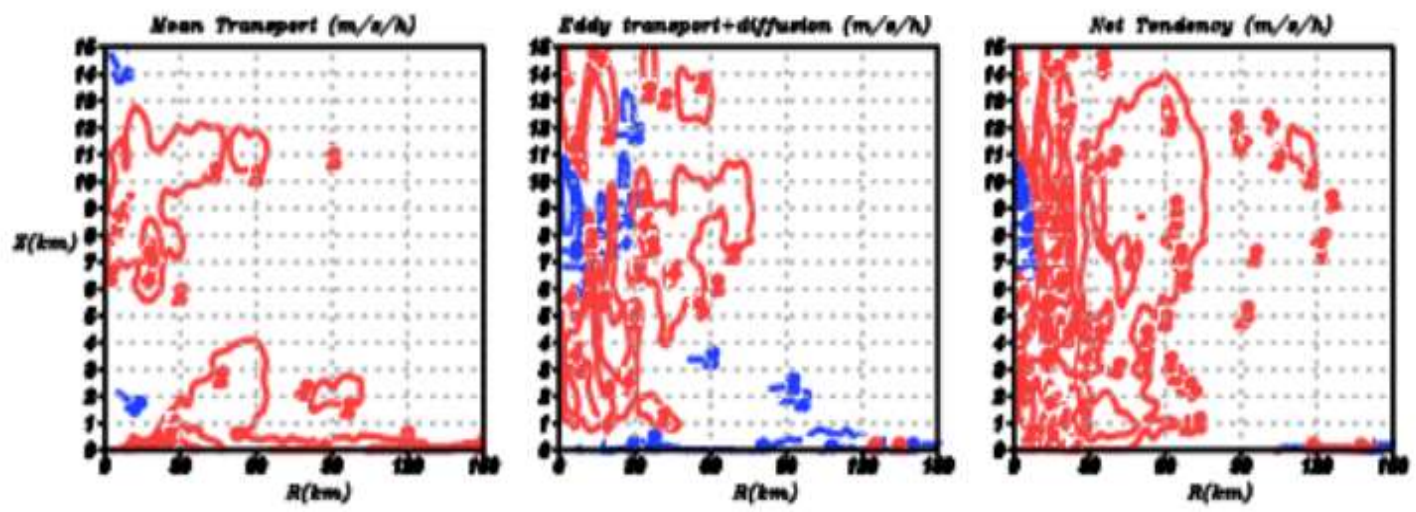

Figure 15. Various terms in the azimuthally averaged tangential wind tendency equation for Hurricane Michael simulation centered at the $37^{\text {th }} \mathrm{hr}$. For all figures, red contours indicate positive values. Blue values indicate negative values azimuthally average, 1 hourly timed averaged, radius height cross-section of a) radial velocity; (m/s), b) tangential velocity $(\mathrm{m} / \mathrm{s})$; c) vertical velocity $(\mathrm{m} / \mathrm{s})$; d) Sum of the mean radial influx of absolute vertical vorticity and the mean vertical advection of the mean tangential momentum $(\mathrm{m} / \mathrm{s} / \mathrm{h})$; e) sum of the eddy radial vorticity flux , the vertical eddy advection of eddy tangential momentum, and sum of the vertical and lateral diffusion terms $(\mathrm{m} / \mathrm{s} / \mathrm{h})$; f) net tendency. Top across a-c. Bottom across d-f. 
During the later stages of RI around the $56^{\text {th }}$ hour, the radial circulation has a strong inflow roughly $1 \mathrm{~km}$ deep in the lower troposphere (Figure 16a). Michael had a defined strong outflow structure with outflow strengths up to $7+\mathrm{m} / \mathrm{s}$ compared to Irma (Figure 14c). The mean tangential wind (Figure 16b) grew stronger and a developed deep vortex passed $16 \mathrm{~km}$ with maximum winds of $45 \mathrm{~m} / \mathrm{s}$ within the hurricane boundary. Figure $16 \mathrm{c}$ shows a defined, strong updraft core on the order of $\sim 1 \mathrm{~m} / \mathrm{s}$ within the eyewall along with strong subsidence within the eye down to the lower levels.

As for the tangential wind momentum budget in later stages of RI (Figure 16d), the budget is still dominated by the mean transport of radial influx of absolute vorticity and the vertical advection of the mean tangential momentum (positively contributed) within the eyewall and hurricane boundary region. Again, the eddy and diffusive terms offset the radial flux of absolute vorticity in the lowest level of the hurricane boundary (Figure 14e and f) as seen in Irma. A significant interesting finding is that the eddy and diffusive terms greatly offsets the spin up process in Michael (Figure 16e). This evidence of hostile wind shear impacting the system continues to intensify in strength. Regardless of moderate to strong shear, impacts, spin up is still prevalent within the eyewall. The overall net tendency (Figure16f) is positive with spin up in the eyewall region. 
A - C
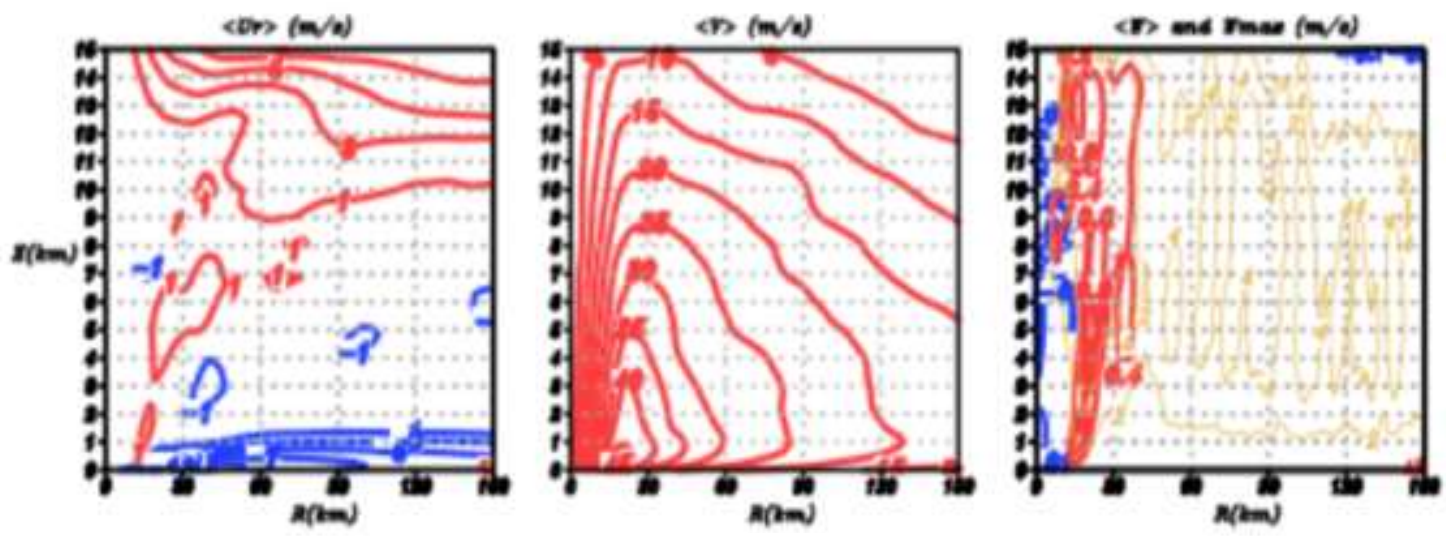

D - E
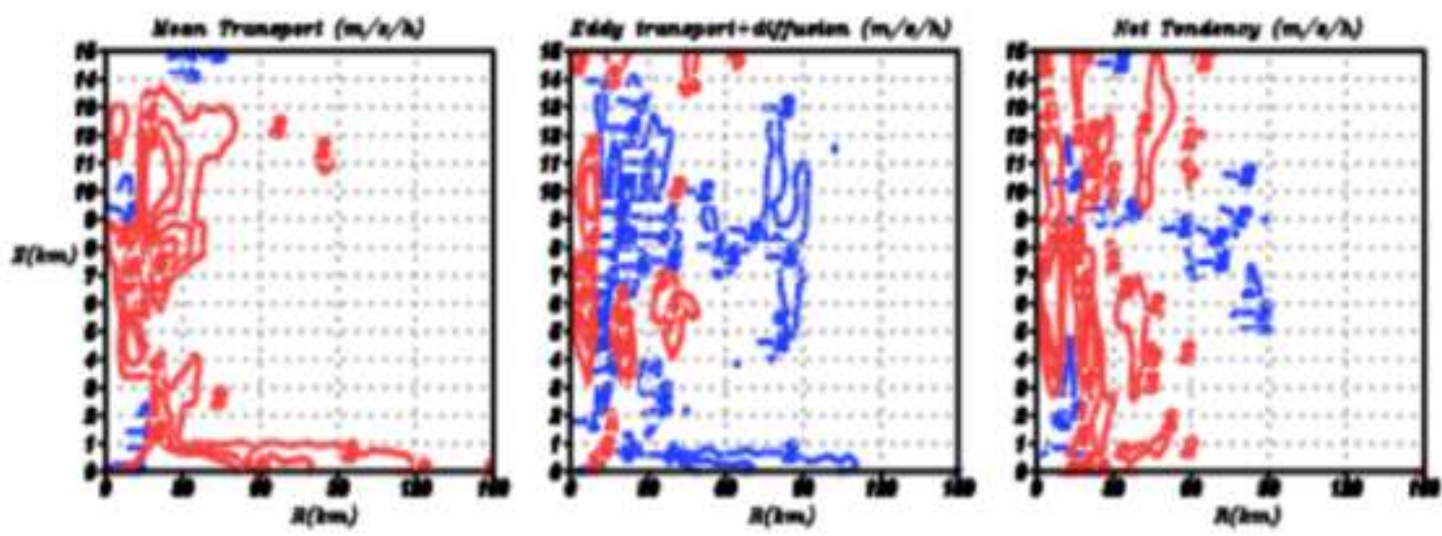

Figure 16. Various terms in the azimuthally averaged tangential wind tendency equation for Hurricane Michael simulation centered at the $56^{\text {th }} \mathrm{hr}$. For all figures, red contours indicate positive values. Blue values indicate negative values azimuthally average, 1 hourly timed averaged, radius height cross-section of a) radial velocity; (m/s), b) tangential velocity $(\mathrm{m} / \mathrm{s})$; c) vertical velocity $(\mathrm{m} / \mathrm{s})$; d) Sum of the mean radial influx of absolute vertical vorticity and the mean vertical advection of the mean tangential momentum $(\mathrm{m} / \mathrm{s} / \mathrm{h}) ;$ e) sum of the eddy radial vorticity flux , the vertical eddy advection of eddy tangential momentum, and sum of the vertical and lateral diffusion terms $(\mathrm{m} / \mathrm{s} / \mathrm{h})$; f) net tendency. Top across a-c. Bottom across d-f. 


\section{CHAPTER 5: CONCLUSIONS AND DISCUSSIONS}

In this study, two TCs (axis-symmetric and asymmetric) were analyzed using forecasts from the HWRF model to study the differences in both inner core structure and large scale environment connection to RI. The study aimed to understand: 1) How and why Hurricane Michael developed as a major hurricane despite hostile environmental conditions, 2) What is the role of eddy vorticity fluxes on TC's intensification processes, and 3) How does the surrounding environment interact with the TC's inner core vortex. For both TCs, the model produced exceptional results reproducing the large scale features as well as tracks and intensity for each case.

The gathered findings show that Irma's axisymmetric RI was highly supported by its favorable (low vertical wind shear, ample symmetrical moist midlevels, marginal SSTs) environmental conditions. Low wind shear allowed for the development of a vertically stacked vortex with deep rotating convective plumes in an environment of abundant vorticity (Figure 11a-b). This is consistent with previous studies (e.g. Hendricks et. al 2014; Yang et al. 2007; Gopalakrishnan et al 2011; Smith 2015). Gopalakrishnan et al. 2019 found that vortical plumes merge and stretch low level vorticity near the boundary layer into small scale anomaly creating a stronger inflow that supports intensification.

The budget analysis of Irma's tangential momentum (Figure 13d-f) revealed that the spin up tangential winds is dominated by the mean transport of radial influx of absolute vorticity and the vertical advection of the mean tangential momentum within the eyewall region. The eddy flux terms along with the diffusive terms (Figure 13e) negatively contributed which showed spin down in the lower levels of the hurricane boundary layer 
and in the upper levels $(7 \mathrm{~km}-15 \mathrm{~km})$ of the eyewall. The overall spin up process takes place solely in the eyewall. These results of Irma are parallel to findings in previous studies that spin up of symmetric TC occurs within the eyewall boundary region (Zhang et al. 2001; Persing et al. 2013; Montgomery and Smith 2014; Gopalakrishnan et al. 2019). Similar results are evident (Figure $14 \mathrm{~d}-\mathrm{f}$ ) in the later stage of RI, except where eddy fluxes created spin down entirely in the eyewall.

Michael's environment had favorable SST, marginally moist midlevels, and hostile wind shear that was highly unfavorable for RI. This environment produced a different inner core structure compared to Irma. Unlike Irma that had an already aligned vortex with vortical cyclonic plumes, strong environmental shear created a highly asymmetric convective pattern located in the downshear left quadrant of Michael (Figure 12a). This illustrates the presence of a titled vortex. Figure 12a shows the strengthening of the vorticity as it propagated into the upshear left region during early RI. The later stage of RI (Figure 12b) shows abundance of vorticity wrapped around the RMW, indicating the merging of the lower and upper level vortices resulting in a symmetrical structure.

The budget analysis of Michael's tangential momentum (Figure 15d-f) during early RI revealed that the spin up tangential winds is dominated by the mean transport of radial influx of absolute vorticity and the vertical advection of the mean tangential momentum and the eddy fluxes within the eyewall region. However, during the later stages of RI, the eddy flux term becomes negative indicating spin down processes, while the mean transport positively contributes to spin up of the eyewall. The overall spin up process takes place within and outside eyewall. These results are different compared to previous 
studies (e.g. Chen and Gopalakrishnan 2015; Leighton et al.2018; Gopalakrishnan et al. 2019) on RI in sheared TCs, where the eddy vorticity flux term was the dominating term during the spin up process in a top-bottom fashion and the mean transport dominates later when the vortex is more aligned. Michael's spin up process suggests a bottom-top fashion indicating that warm ocean fluxes may play a significant role in its resilience to combat hostile wind shear during the RI process. 


\section{REFERENCES}

Bevin II, J.L., Berg, R., Hagen, A., 2019: National Hurricane Center Tropical Cyclone Report: Hurricane Michael. National Hurricane Center,1-86. https://www.nhc.noaa.gov/data/tcr/AL142018_Michael.pdf

Bhalachandran, S., Nadimpalli, R., Osuri, K. K., Marks Jr., F. D., Gopalakrishnan, S., Subramanian, S., Mohanty, U. C. and Niyogi, D., 2019: On the processes influencing rapid intensity changes of tropical cyclones over the Bay of Bengal. Scientific Reports, 9, 3382

Bhatia, K.T. and D.S Nolan, 2013: Relating the skill of the tropical cyclone intensity forecast to the synoptic movement. Wea. Forecasting, 28, 961-980.

Biswas M. K., S. Abarca, L. Bernardet, I. Ginis, E. Grell, M. Iacono, E. Kalina, B. Liu, Q. Liu, T. Marchok, A. Mehra, K. Newman, J. Sippel, V. Tallapragada, B. Thomas, W. Wang, H. Winterbottom, and Z. Zhang, 2018: Hurricane Weather Research and Forecasting (HWRF) Model: 2018 Scientific Documentation, Available at https://dtcenter.org/HurrWRF/users/docs/index.php

Black, P. G., 1983: Ocean temperature changes induced by tropical cyclones. Ph.D. dissertation, The Pennsylvania State University, 278 pp. [Available from The Pennsylvania State University, University Park, PA 16802.]

Byers, H. R., 1944: General Meteorology. McGraw-Hill, 645 pp.

Cangialosi, J.P., Latto, A.S., Berg, R., 2018: National Hurricane Center Tropical Cyclone Report: Hurricane Irma. National Hurricane Center, 1-111. https://www.nhc.noaa.gov/data/tcr/AL112017_Irma.pdf

Chen, H., and S. Gopalakrishnan, 2015: A study on the asymmetric rapid intensification of Hurricane Earl (2010) using the HWRF system. J. Atmos. Sci., 72, 531550.doi.org/10.1175/JAS-D-14-0097.1

DeMaria, M., and J. Kaplan, 1994: Sea surface temperature and the maximum intensity of Atlantic tropical cyclones. J. Climate, 7, 1324-1334.doi.org/10.1175/15200442(1994)007<1324:SSTATM>2.0.CO;2 
DeMaria, M., 1996: The effect of vertical shear on tropical cyclone intensity change. $J$. Atmos. Sci., 53, 2076-2087. doi.org/10.1175/15200469(1996)053<2076:TEOVSO>2.0.CO;2

Dengler, K., 1997: A numerical study of the effects of land proximity and changes in sea surface temperature on hurricane tracks. Quart. J. Roy. Meteor. Soc., 123, $1307-$ 1321. doi.org/10.1002/qj.49712354109

Eastin, M. D., W. M. Gray, and P. G. Black, 2005a: Buoyancy of convective vertical motions in the inner core of intense hurricanes. Part I: General statistics. Mon. Wea. Rev., 133, 188-208.doi.org/10.1175/MWR-2848.1

Eastin, M. D., W. M. Gray, and P. G. Black, 2005b: Buoyancy of convective vertical motions in the inner core of intense hurricanes. Part II: Case studies. Mon. Wea. Rev., 133, 209-227.doi.org/10.1175/MWR-2849.1

Ge, X., Li, T., and Peng, M.: Effects of Vertical Shears and Midlevel Dry Air on Tropical Cyclone Developments, J. Atmos. Sci., 70, 3859-3875.doi:10.1175/JAS-D-13-066.1, 2013

Gray, W. M., 1968: Global view of the origin of tropical disturbances and storms. Mon. Wea. Rev., 96, 669-700.doi.org/10.1175/15200493(1968)096<0669:GVOTOO>2.0.CO;2

Gopalakrishnan, S. G., Marks, F., Zhang, X. J., Bao, J. W., Yeh, K. S. and Atlas, R., 2011: The Experimental HWRF System: A Study on the Influence of Horizontal Resolution on the Structure and Intensity Changes in Tropical Cyclones Using an Idealized Framework. Mon. Wea. Rev., 139, 1762-1784.

Gopalakrishnan, Sundararaman \& Osuri, Krishna \& Marks, Frank \& Mohanty, U C. (2019): An inner-core analysis of the axisymmetric and asymmetric intensification of tropical cyclones: Influence of shear. Mausam., 70. 667-690.

Hendricks, E. A., Montgomery, M. T. and Davis, C. A., 2004: The role of "vortical" hot towers in the formation of tropical cyclone Diana (1984). J. Atmos. Sci., 61, 12091232. 
Hendricks, E. A., Peng, M. S., Fu, B., and Li, T.: Quantifying Environmental Control on Tropical Cyclone Intensity Change, Mon. Weather Rev., 138, 3243-3271. doi:10.1175/2010MWR3185.1, 2010

Kaplan, J., and M. DeMaria, 2003: large scale characteristics of rapidly intensifying tropical cyclones in the North Atlantic basin. Wea. Forecasting, 18, 10931108.doi.org/10.1175/1520-0434(2003)018<1093:LCORIT>2.0.CO;2

Kaplan, J., M. DeMaria, and J. A. Knaff, 2010: A revised tropical cyclone rapid intensification index for the Atlantic and eastern North Pacific basins. Wea. Forecasting, 25, 220-241.doi.org/10.1175/2009WAF2222280.1

Kaplan, J., and Coauthors, 2015: Evaluating environmental impacts on tropical cyclone rapid intensification predictability utilizing statistical models. Wea. Forecasting, 30, 1374-1396.doi.org/10.1175/WAF-D-15-0032.1

Kossin, J., and W. H. Schubert, 2001: Mesovortices, polygonal flow patterns, and rapid pressure falls in hurricane-like vortices. J. Atmos. Sci., 58, 21962209.doi.org/10.1175/1520-0469(2001)058<2196:MPFPAR>2.0.CO;2

Leighton, H., Gopalakrishnan, S., Zhang, J. A., Rogers, R. F., Zhang, Z. and Tallapragada, V., 2018: Azimuthal distribution of deep convection, environmental factors and tropical cyclone rapid intensification: A perspective from HWRF ensemble forecasts of Hurricane Edouard (2014)", J. Atmos. Sci., 75, 275-295.

Marks, F. D., Jr., and Coauthors, 1998: Landfalling tropical cyclones: Forecast problems and associated research opportunities. Report of the Fifth Prospectus Development Team to the U.S. Weather Research Program. Bull. Amer. Meteor. Soc., 79, 305-323.

Merrill, R. T., 1988: Environmental influences on hurricane intensification. J. Atmos. Sci., 45, 1678-1687.doi.org/10.1175/1520-0469(1988)045<1678:EIOHI>2.0.CO;2

Molinari, J., P. Dodge, D. Vollaro, K. L. Corbosiero, and F. Marks Jr., 2006: Mesoscale aspects of the downshear reformation of a tropical cyclone. J. Atmos. Sci., 63, 341354.doi.org/ 10.1175/JAS3591.1

Molinari, J., and D. Vollaro, 2010: Rapid intensification of a sheared tropical storm. Mon. Wea. Rev., 138, 3869-3885.doi.org/10.1175/2010MWR3378.1 
Montgomery, M. T. and Smith, R. K., 2014, "Paradigms for tropical cyclone intensification", Australian Meteorological and Oceanographic Journal, 64, 37-66.

Nguyen, S., Smith, R. and Montgomery, M., 2008, "Tropical-cyclone intensification and predictability in three dimensions", Q. J. R. Meteo. Soc., 134, 563-582.

Palmén, E. H., 1948: On the formation and structure of tropical cyclones. Geophysical, 3, 26-38.

Persing, J., Montgomery, M. T., McWilliams, J. C. and Smith, R. K., 2013: Asymmetric and axisymmetric dynamics of tropical cyclones. Atmospheric Chemistry and Physics, 13, 12299-12341.

Rappaport, E. N., and Coauthors, 2009: Advances and challenges at the National Hurricane Center. Wea. Forecasting, 24, 395-419.

Riemer, M., Montgomery, M. T. and Nicholls, M. E., 2010: A new paradigm for intensity modification of tropical cyclones: Thermodynamic impact of vertical wind shear on the inflow layer. Atmospheric Chemistry and Physics., 10, 3163-3188.

Rios-Berrios, R., R.D. Torn, and C.A. Davis, 2016: An ensemble approach to investigate tropical cyclone intensification in a sheared environment. Part I: Katia (2011). J. Atmos. Sci., 73, 71-93.

Schubert, W. H., and J. J. Hack, 1982: Inertial stability and tropical cyclone development. J. Atmos. Sci., 39, 1687-1697.doi.org/10.1175/15200469(1982)039<1687:ISATCD>2.0.CO;2

Shay, L. K., G. J. Goni, and P. G. Black, 2000: Effects of a warm oceanic feature on Hurricane Opal. Mon. Wea. Rev., 128, 1366-1383.doi.org/10.1175/15200493(2000)128<1366:EOAWOF>2.0.CO;2

Shen, B.-W., W.-K. Tao, W. K. Lau, and R. Atlas, 2010: Predicting tropical cyclogenesis with a global mesoscale model: Hierarchical multiscale interactions during the formation of Tropical Cyclone Nargis (2008). J. Geophys. Res., 115, D14102, doi:10.1029/2009JD013140. 
Smith, R. K. and Montgomery, M. T., 2015: Toward clarity on understanding tropical cyclone intensification", J. Atmos. Sci., 72, 3020-3031.

Tang, B. and Emanuel, K., 2010: Midlevel ventilation's constraint on tropical cyclone intensity. J. Atmos. Sci., 67, 1817-1830.

Tang, B. and Emanuel, K., 2012: Sensitivity of tropical cyclone intensity to ventilation in an axisymmetric model, J. Atmos. Sci., 69, 2394-2413.

Tao, D. and Zhang F., 2014: Effect of environmental shear, sea surface temperature, and ambient moisture on the formation and predictability of tropical cyclones: An ensemble mean perspective, J. Adv. Model. Earth Syst., 6, 384-404. doi:10.1002/2014MS000314.

Willoughby, H. E., J. A. Clos, and M. G. Shoreibah, 1982: Concentric eyewalls, secondary wind maxima, and the evolution of the hurricane vortex. J. Atmos. Sci., 39, 395-411.doi.org/10.1175/1520-0469(1982)039<0395:CEWSWM>2.0.CO;2

Wu, L., Su, H., Fovell, R. G., Wang, B., Shen, J. T., Kahn, B. H., Hristova-Veleva, S. M., Lambrigtsen, B. H., Fetzer, E. J., and Jiang, J. H, 2012 : Relationship of environmental relative humidity with North Atlantic tropical cyclone intensity and intensification rate. Geophys. Res. Lett., 39, L20809. doi:10.1029/2012GL053546.

Yang, B., Wang, Y. Q. and Wang, B., 2007: The effect of internally generated inner-core asymmetries on tropical cyclone potential intensity. J.Atmos.Sci., 64, 1165-1188.

Zhang, D. L., Liu, Y. B. and Yau, M. K., 2001: A multiscale numerical study of Hurricane Andrew (1992). Part IV: Unbalanced flows. Mon.Wea.Rev., 129, 92-107.

Zhang, F., and D. Tao, 2013: Effects of vertical wind shear on the predictability of tropical cyclones. J. Atmos. Sci., 70, 975-983.doi.org/10.1175/JAS-D-12-0133.1 